\title{
A Compact Basis for Reliable Fast Frequency Sweep via the Reduced-Basis Method
}

\author{
Valentín de la Rubia and Michal Mrozowski
}

\begin{abstract}
A reliable reduced-order model (ROM) for fast frequency sweep in time-harmonic Maxwell's equations by means of the reduced-basis method is detailed. Taking frequency as a parameter, the electromagnetic field in microwave circuits does not arbitrarily vary as frequency changes, but evolves on a very low-dimensional manifold. Approximating this low-dimensional manifold by a low dimension subspace, namely, reduced-basis space, gives rise to an ROM for fast frequency sweep in microwave circuits. This avoids carrying out time-consuming finite-element analysis for each frequency in the band of interest. The behavior of the solutions to Maxwell's equations as a function of the frequency parameter is studied and highlighted. As a result, a compact reduced-basis space for efficient model-order reduction is proposed. In this paper, the reduced-basis space is composed of two parts: 1) eigenmodes hit in the frequency band of interest, which form an orthogonal, fundamental set that describes the natural oscillating dynamics of the electromagnetic field and 2) whatever else electromagnetic fields, sampled in the frequency band of interest, that are needed to achieve convergence in the reduced-basis approximation. The reduced-basis method aims not only to find out a reduced-basis space in an efficient way, but also to certify the reliability of the approximation carried out. Emphasis is placed on a fast evaluation of the ROM error measure and on providing a reliable convergence criterion. This approach is applied to both narrowband resonating structures and wideband nonresonanting devices in order to show the capabilities of the method in real-life applications.
\end{abstract}

Index Terms - Computer-aided engineering, design automation, error analysis, finite-element methods, Galerkin method, microwave circuits, reduced basis methods, reduced-order systems.

\section{INTRODUCTION}

$\mathbf{I}$ NCREASING deployment of telecommunication services is urging microwave industry to speed up product development time, where not only prototyping and final tuning steps in the production line have to be reduced, but also the simulation time to get an actual electrical design by means of computer-aided design (CAD). A significant cost reduction can be achieved in prototyping and tuning steps if electrical designs are optimized to do so, in addition to satisfying electrical specifications. Performing robust electrical designs has thus become of paramount importance, and powerful numerical techniques capable of predicting electromagnetic behavior are at the heart of this robust design.

When developing an electrical design, several geometrical parameters, say a few tens, need to be optimized via CAD. The change in the geometry can be large from the starting point in the optimization until the final design is obtained. A large amount of different geometry simulations may be required. However, to speed up each geometry simulation, model-order reduction techniques can be of help. Model-order reduction consists of replacing a rather complex mathematical model by a much simpler approximated one that still maintains certain aspects of the original model [1]. Instead of performing time-consuming analysis, such as finite-element (FEM) or boundary-element (BEM) analysis, for each frequency in the band of interest, a fast frequency sweep can be carried out by means of a reduced-order model (ROM). In order for this ROM to be of actual interest for CAD rather than for academic interest, several issues need to be addressed. First, both the model-order reduction generation process and the evaluation of the ROM itself has to be considered, since each new geometry simulation implies a new model-order reduction starting from scratch. Second, model-order reduction should be a completely automatic process, where no human interaction can be afforded, as this fast frequency sweep is held within a CAD optimization loop. Finally, ROM has to be reliable and its accuracy has to be certified to guarantee that the optimization routine is not misled by inaccurate ROMs. We focus on all this in this paper.

There is an intensive research activity on model-order reduction. In this sense, there are two different approaches, namely, moment-matching techniques and singular-value decomposition (SVD) strategies. The former is based on expanding the complex mathematical model into a Taylor series and getting an ROM by matching the moments or Taylor coefficients to a Padé approximant. The latter requires a large amount of field solutions at different frequencies, and then, SVD is applied to extract the fundamental dynamics in the original system by means of the dominant singular vectors. An ROM is obtained by applying Galerkin projection to the original system onto the subspace spanned by the dominant singular vectors. 
However, each of these approaches can be further combined into the other, namely, not only Taylor series in multiple expansion points can be considered to improve convergence in the moment-matching framework, but also derivatives in field solutions can uplift the field solution data for analysis in SVD-based model-order reduction. The following papers deal with these strategies [2]-[10].

Model-order reduction techniques have been widely used in computational electromagnetics. Reference [11] minimizes the computational complexity of scattering problems in Electromagnetics applying an ROM to the electric-field volume integral equation method. Eigenmode expansion is considered in [12] for model-order reduction, since the excited fields can be approximately described by a judicious selection of a few dominant eigenmodes. A full set of eigenmodes is proposed as projection space in [13] and [14] to extract the dominant behavior in the device transfer function. Fast frequency sweep in domain decomposition approaches has been proposed in [15] and [16]. A fast frequency sweep for infinite array antennas based on SVD-Krylov model-order reduction is proposed in [17]. Reference [18] addresses an ROM for fast frequency sweep in microwave devices where frequency-dependent modal field patterns are considered on the excitation ports. Remarkably, the problem in [18] is formulated in such a way that eigenresonances are not picked in the basis construction. A physical-based convergence criterion for fast frequency sweep in FEM analysis is proposed in [19], where energy conservation constraints are imposed to the ROM.

Considering frequency as a parameter, the FEM formulation for time-harmonic Maxwell's equations we aim to solve turns into a frequency-dependent variational problem. If we do not consider the similarities in the field solutions along the frequency band of interest, we can solve for this frequency-dependent variational problem in a completely independent way, where each field solution is obtained from scratch for each frequency point of analysis. As a result, the frequency-dependent variational problem is solved for each frequency of interest as a standard FEM problem, which yields to a rather time-consuming approach. However, if we put all these field solutions together and determined how linearly independent they are, we would realize that all these field solutions stay within a manifold of low dimension, induced by the parameter dependence. Finding a linear space of small dimension, namely, reduced-basis space, approximating this manifold is the idea behind the reduced-basis method [20]-[24]. This reduced-basis space, with a small dimension, will be the space where the solution to the frequency-dependent variational problem is sought for, giving rise to a small-size variational problem. As a result, the frequency-dependent variational problem can be solved in a fast frequency sweep model-order reduction setting.

Contrary to what has been previously done in the reduced-basis method context, in this paper, we take a closer look at the electromagnetic-field behavior as a function of the frequency parameter and identify what is the characteristic of the best approximation basis to span the reduced-basis space. As a result, we propose a new compact reduced-basis space that is composed of two parts: 1) eigenmodes hit in the frequency band of interest, which form an orthogonal, fundamental set that describes the natural oscillating dynamics of the electromagnetic field and 2) samples of the electromagnetic field, evaluated at certain points in the frequency band of interest, that span whatever else that is needed to achieve convergence in the reduced-basis approximation.

In the context of FEM computations, it should be pointed out that it is unrealistic to compute hundreds or thousands of eigenmodes from FEM analysis, especially in large FEM systems. However, in the technique we are proposing, just a few eigenmodes have to be computed, namely, the ones that are hit in the frequency band of interest.

By the same token, the ROM needs to be certified and an a posteriori error analysis is developed. Different error estimators have been proposed [17], [22], [23], [25], [26] and some of them are based on the inf-sup constant in the frequency-dependent variational problem [27]. However, whenever a resonance is hit in the frequency band of interest, which is normally the case in microwave circuits, since most devices involve discontinuities that often result in resonances, these inf-sup constant-based error estimators are no longer valid. We consider this problem and propose an alternative to certify the accuracy in the ROM. This is the second new contribution of this paper that we would like to highlight. In addition to the eigenmodes hit in the frequency band of analysis, a special emphasis is placed in identifying the most linearly independent in-band electromagnetic-field solutions that can be collected to span the reduced-basis space.

As far as comparison purposes are concerned, we provide a comparison of the proposed approach with the optimal model-order reduction technique, namely, proper orthogonal decomposition (POD) [20], [21]. It is well-known that POD provides the optimal reduction results, as it measures the linear independence found within the parameter-dependent field solutions, and therefore states the maximum compression reduction. However, POD is only of academic interest in practice, since it requires a postprocessing of all field solutions in the parameter space, thus requiring a very fine frequency sampling in the band of interest by means of FEM analyses, which cannot be afforded in practical industrial applications. Nevertheless, this is a fair comparison, since it actually states where the proposed setting stays with respect to this utopian optimal ROM.

Prior to going through all the details, we would like to claim that the reduced-basis method we are proposing makes it possible to solve for the electromagnetic behavior of a circuit by solving a small system of equations when a fast frequency sweep is carried out. However, this reduction does not come for free. This is due to the fact that the reduced-basis method needs to build a reduced-basis space up first in order to compute the ROM. This computation is carried out offline and does require FEM analyses. However, the number of FEM analyses needed to build up the ROM is just a few analyses such as the order of the ROM.

This paper is organized as follows. In Section II, we review the time-harmonic Maxwell's equations in variational form and solve for the electromagnetic field in order to show its 
frequency behavior. Section III deals with the reduced-basis method, where the ingredients to carry out a reliable model-order reduction for fast frequency sweep in electromagnetics are detailed. Section IV shows numerical examples and illustrates the capabilities of the proposed approach as well as its accuracy. Finally, in Section V, we comment on the conclusions.

\section{Problem Statement}

Maxwell's equations govern the electromagnetic behavior in a given structure. Applying the Fourier transform to these, one can find the fields in the transform domain $i \omega$. They are

$$
\begin{aligned}
& \nabla \times E=-i \omega \mu H \text { in } \Omega \\
& \nabla \times H=i \omega \varepsilon E \text { in } \Omega \\
& n \times E=0 \text { on } \Gamma_{\mathrm{PEC}} \\
& n \times H=0 \text { on } \Gamma_{\mathrm{PMC}} \\
& n \times H=J \text { on } \Gamma
\end{aligned}
$$

where $\Omega \subset \mathbb{R}^{3}$ is a source-free sufficiently smooth bounded domain and $n$ is the unit outward normal vector on the boundary $\partial \boldsymbol{\Omega}$ of $\boldsymbol{\Omega}$; the boundary $\partial \boldsymbol{\Omega}$ of $\boldsymbol{\Omega}$ is divided into perfect electric conductor (PEC), perfect magnetic conductor (PMC), and ports, i.e., $\partial \Omega=\Gamma_{\mathrm{PEC}} \cup \Gamma_{\mathrm{PMC}} \cup \Gamma . E$ and $H$ are the electric and magnetic vector fields, $\varepsilon$ and $\mu$ are, respectively, the permittivity and permeability of the medium, which is assumed to be lossless, and the tangential vector field $J$ is the excitation current at the ports. Time-harmonic Maxwell's equations can be written in a classical weak formulation over an appropriate admissible function space $\mathcal{H}$, viz.,

Find $E \in \mathcal{H}$ such that

$$
a(E, v)=f(v) \quad \forall v \in \mathcal{H} \text {. }
$$

The bilinear form being

$$
a(E, v)=\int_{\Omega}\left(\frac{1}{\mu} \nabla \times E \cdot \nabla \times v-\omega^{2} \varepsilon E \cdot v\right) d x
$$

and the linear form

$$
f(v)=i \omega \int_{\partial \Omega} J \cdot v d s=i \omega \int_{\Gamma} J \cdot v d s .
$$

Here, the admissible space $\mathcal{H}$ stays in the Hilbert space $H(\operatorname{curl}, \Omega)$ defined by

$$
H(\operatorname{curl}, \Omega)=\left\{u \in L^{2}\left(\Omega, \mathbb{C}^{3}\right) \mid \nabla \times u \in L^{2}\left(\Omega, \mathbb{C}^{3}\right)\right\} .
$$

$\mathcal{H}$ should consider the boundary condition (1c), namely,

$$
\mathcal{H}=\left\{u \in H(\operatorname{curl}, \Omega) \mid n \times u=0 \text { on } \Gamma_{\mathrm{PEC}}\right\}
$$

Let us refer to the trace spaces, namely,

$$
\begin{aligned}
& H^{-1 / 2}(\operatorname{Div}, \partial \Omega)=\{n \times u \text { on } \partial \Omega \mid u \in H(\operatorname{curl}, \Omega)\} \\
& H^{-1 / 2}(\operatorname{curl}, \partial \Omega)=\{n \times u \times n \text { on } \partial \Omega \mid u \in H(\operatorname{curl}, \Omega)\}
\end{aligned}
$$

and point out that they are dual to each other with the following duality pairing:

$$
\langle u, v\rangle=\int_{\partial \Omega} u \cdot v d s
$$

where $u \in H^{-1 / 2}(\operatorname{Div}, \partial \Omega)$ and $v \in H^{-1 / 2}(\operatorname{curl}, \partial \Omega)$. In this sense, we can now point out that $J$ belongs to $H^{-1 / 2}$ (Div, $\partial \Omega$ ). We refer to [28] and [29] for a thorough explanation of all these spaces.

The purpose of this section is to show the frequency behavior of the electromagnetic field, as well as state some results that will be further discussed in this paper. In order to do so, we solve for Maxwell's equations from a functional analysis point of view. The Riesz representation theorem and the Fredholm alternative will be applied. We refer to [30]-[32] for a detailed explanation of these fundamental results. Spectral theory for self-adjoint compact operators will be used. To this end, a compact operator needs to be identified within Maxwell's equations, and this is done by introducing a Helmholtz decomposition in the solution space $\mathcal{H}$.

Let us proceed with the derivation to identify the frequency behavior of the electromagnetic field, based on the Fredholm alternative and spectral theory of self-adjoint compact operators. In order to show some structure in the solution to the variational problem (2), we introduce the Helmholtz decomposition [33]

$$
\mathcal{H}=\mathcal{H}(\operatorname{curl} 0, \Omega) \oplus \mathcal{V}
$$

where

$$
\begin{aligned}
\mathcal{H}(\operatorname{curl} 0, \Omega) & =\{u \in \mathcal{H} \mid \nabla \times u=0\} \\
\mathcal{V} & =\left\{u \in \mathcal{H} \mid(\varepsilon u, v)_{L^{2}(\Omega)}=0 \forall v \in \mathcal{H}(\operatorname{curl} 0, \Omega)\right\}
\end{aligned}
$$

$(\cdot, \cdot)_{L^{2}(\Omega)}$ is the inner product in $L^{2}\left(\Omega, \mathbb{C}^{3}\right) . \mathcal{H}(\operatorname{curl} 1, \Omega)$ denotes the null space of the curl operator, whereas $\mathcal{V}$ stands for its orthogonal complement within the solution space $\mathcal{H}$ in the following inner product:

$$
(u, v)_{\mu, \varepsilon}=\left(\frac{1}{\mu} \nabla \times u, \nabla \times v\right)_{L^{2}(\Omega)}+(\varepsilon u, v)_{L^{2}(\Omega)} .
$$

It should be noted that both $\mathcal{H}(\operatorname{curl} 10, \Omega)$ and $\mathcal{V}$ spaces satisfy the PEC boundary condition on $\Gamma_{\mathrm{PEC}}$.

This splitting (9) suggests the following decomposition in the trace space $H^{-1 / 2}$ (Div, $\partial \boldsymbol{\Omega}$ ), namely:

$$
H^{-1 / 2}(\operatorname{Div}, \partial \Omega)=\mathcal{U} \oplus \mathcal{W}
$$

where

$$
\begin{aligned}
& \mathcal{W}=\left\{j \in H^{-1 / 2}(\text { Div }, \partial \Omega) \mid\right. \\
& \quad\langle j, n \times \bar{v} \times n\rangle=0 \quad \forall v \in \mathcal{H}(\operatorname{cur} 10, \Omega)\} \\
& \mathcal{U}=H^{-1 / 2}(\operatorname{Div}, \partial \Omega) \backslash \mathcal{W} \cup\{0\}
\end{aligned}
$$

and $\bar{v}$ stands for complex conjugate of $v$.

Now, we solve for the variational problem (2) using the decompositions (9) and (12), namely, the following splittings are then used $E=E_{0}+e, E_{0} \in \mathcal{H}(\operatorname{curl} 0, \Omega), e \in \mathcal{V}$, and $J=J_{0}+j, J_{0} \in \mathcal{U}, j \in \mathcal{W}$. Replacing these splittings in (2) yields

$$
\begin{aligned}
\int_{\Omega}\left(\frac{1}{\mu} \nabla \times e \cdot \nabla \times \bar{v}-\omega^{2} \varepsilon\left(E_{0}\right.\right. & +e) \cdot \bar{v}) d x \\
& =i \omega \int_{\Gamma}\left(J_{0}+j\right) \cdot \bar{v} d s
\end{aligned}
$$


for all $v \in \mathcal{H}$, where we have deliberately changed $v$ by its complex conjugate $\bar{v}$ to bring inner products in place. First, we test (14) in $\mathcal{H}($ curlo, $\Omega$ ), which results in

$$
-\omega^{2} \int_{\Omega} \varepsilon E_{0} \cdot \bar{v} d x=i \omega \int_{\Gamma} J_{0} \cdot \bar{v} d s \quad \forall v \in \mathcal{H}(\operatorname{curl} 1, \Omega) .
$$

The Riesz representation theorem [30] guarantees unique solution to (15). Let us denote $F_{0} \in \mathcal{H}(\operatorname{curl} 0, \Omega)$ as the Riesz representative in this problem (15) for $i w E_{0}$. Then

$$
E_{0}=\frac{1}{i w} F_{0} .
$$

Considering that (15) is solvable, (14) simplifies to

$$
\int_{\Omega}\left(\frac{1}{\mu} \nabla \times e \cdot \nabla \times \bar{v}-\omega^{2} \varepsilon e \cdot \bar{v}\right) d x=i \omega \int_{\Gamma} j \cdot \bar{v} d s
$$

for all $v \in \mathcal{H}$. Second, we test (17), and implicitly (14), in $\mathcal{V}$, resulting in the following variational problem in $\mathcal{V}$ that remains to be solved:

Find $e \in \mathcal{V}$ such that $\int_{\Omega}\left(\frac{1}{\mu} \nabla \times e \cdot \nabla \times \bar{v}-\omega^{2} \varepsilon e \cdot \bar{v}\right) d x$

$$
=i \omega \int_{\Gamma} j \cdot \bar{v} d s \quad \forall v \in \mathcal{V} .
$$

In order to show solvability in (18), we introduce an equivalent inner product to (11) in $\mathcal{V}$ [33], namely,

$$
(u, v)_{*}=\left(\frac{1}{\mu} \nabla \times u, \nabla \times v\right)_{L^{2}(\Omega)} .
$$

Now, the variational problem (18) can be rewritten as

$$
(e, v)_{*}-\omega^{2}(\varepsilon e, v)_{L^{2}(\Omega)}=i \omega\langle j, n \times \bar{v} \times n\rangle \quad \forall v \in \mathcal{V} .
$$

Next, we use the Riesz representation theorem. For fixed $e \in L^{2}\left(\Omega, \mathbb{C}^{3}\right)$, the mapping $v \mapsto(v, \varepsilon e)_{L^{2}(\Omega)}$ for all $v \in \mathcal{V}$ is a linear and bounded functional on $\mathcal{V}$, and by the Riesz representation theorem, there exists a unique $g_{e} \in \mathcal{V}$ with $(v, \varepsilon e)_{L^{2}(\boldsymbol{\Omega})}=\left(v, g_{e}\right)_{*}$ for all $v \in \mathcal{V}$. Then, we can define the operator $\tilde{K}: L^{2}\left(\Omega, \mathbb{C}^{3}\right) \rightarrow \mathcal{V}$ that maps $e$ to its Riesz representative $g_{e}$, namely, $e \mapsto \tilde{K} e=g_{e}$. This operator is bounded and linear [33]. By the same token, as a result of the Helmholtz decomposition (9), $\mathcal{V}$ is compactly embedded in $L^{2}\left(\Omega, \mathbb{C}^{3}\right)$ [33]. If we denote by $M: \mathcal{V} \rightarrow L^{2}\left(\Omega, \mathbb{C}^{3}\right)$ the compact embedding operator, we can then show that $K=$ $\tilde{K} \circ M$ is a compact operator. This first result is of paramount importance in our reasoning, because it allows us to use the Fredholm alternative, which is applicable to compact operators and cannot be used for Maxwell's equations in their original form.

Once again, we use the Riesz representation theorem in a similar way. For fixed $j \in \mathcal{W}$, the mapping $v \mapsto\langle j, n \times v \times n\rangle$ for all $v \in \mathcal{V}$ is a linear and bounded functional on $\mathcal{V}$, and then by the Riesz representation theorem, there exists a unique $g_{j} \in \mathcal{V}$ with $\langle\bar{j}, n \times v \times n\rangle=\left(v, g_{j}\right)_{*}$ for all $v \in \mathcal{V}$. We can define the operator $\tilde{L}: \mathcal{W} \rightarrow \mathcal{V}$ that maps $j$ to its Riesz representative $g_{j}$, namely, $j \mapsto \tilde{L} j=g_{j}$.

As a result, (20) can be written as

$$
(e, v)_{*}-\omega^{2}(K e, v)_{*}=i \omega(\tilde{L} j, v)_{*} \quad \forall v \in \mathcal{V} \text {. }
$$

Since this holds for all $v \in \mathcal{V}$, this equation is equivalent to

$$
e-\omega^{2} K e=i \omega \tilde{L} j \text { in } \mathcal{V} \text {. }
$$

At this point, we have arrived at an operator form of Maxwell's equations with operator $K$ having the properties needed in order to use the Fredholm theorem and the spectral theory. The operator $K$ is not only compact, but also self-adjoint with respect to the bilinear form $\langle u, v\rangle_{*}=(u, \bar{v})_{*}$ in $\mathcal{V}$. Indeed, $\langle K u, v\rangle_{*}=(K u, \bar{v})_{*}=(\varepsilon u, \bar{v})_{L^{2}(\Omega)}$, and since this is symmetric in $u$ and $v$, we get $\langle K u, v\rangle_{*}=\langle u, K v\rangle_{*}$. The Fredholm theorem [31], [32] states that (22) is solvable for those $j \in \mathcal{W}$, such that $\tilde{L} j$ is orthogonal to the null space of $I-\omega^{2} K$ with respect to $\langle\cdot, \cdot\rangle_{*}$. Then, the Fredholm alternative further applies, namely, either

$$
e=i \omega \sum_{n=1}^{\infty} \frac{A_{n}}{1-\frac{\omega^{2}}{\omega_{n}^{2}}} e_{n} \quad \text { if } \omega^{2} \neq \omega_{n}^{2}
$$

where

$$
A_{n}=\left\langle\tilde{L} j, \bar{e}_{n}\right\rangle_{*}=\left(\tilde{L} j, e_{n}\right)_{*}=\left\langle j, n \times \bar{e}_{n} \times n\right\rangle
$$

or

$$
e=i \omega \sum_{\omega_{n}^{2}=\omega^{2}} a_{n} e_{n}+i \omega \sum_{\omega_{n}^{2} \neq \omega^{2}} \frac{A_{n}}{1-\frac{\omega^{2}}{\omega_{n}^{2}}} e_{n} \quad \text { if } \omega^{2}=\omega_{n}^{2}
$$

where

$$
\left\langle\tilde{L} j, \bar{e}_{n}\right\rangle_{*}=\left\langle j, n \times \bar{e}_{n} \times n\right\rangle=0 \text { for } \omega_{n}^{2}=\omega^{2}
$$

where $a_{n}$ are arbitrary constants, since the solution is not unique whenever $\omega^{2}=\omega_{n}^{2}$, and $A_{n}$ are defined as in (24). Solutions to the homogeneous problem in (22), namely,

Find $\left(e_{n}, \omega_{n}^{2}\right) \in \mathcal{V} \times \mathbb{R}^{+}$such that

$$
\int_{\Omega}\left(\frac{1}{\mu} \nabla \times e_{n} \cdot \nabla \times v-\omega_{n}^{2} \varepsilon e_{n} \cdot v\right) d x=0 \quad \forall v \in \mathcal{V}
$$

are considered as the representation basis in the solution for the nonhomogeneous problem (22). Eigenvalues $\omega_{n}^{2}$ have finite multiplicity; they are infinitely many and accumulate at infinity. If $e_{n}$ solves (27), then its complex conjugate also does. As a result, the eigenfunctions $e_{n}$ are real-valued functions and, in particular, $\bar{e}_{n}$ can be replaced by $e_{n}$ in (24) and (26). In addition, the set of eigenfunctions $\left\{e_{n} \mid n \in \mathbb{N}\right\}$ forms a complete orthonormal system in $\mathcal{V}$ [33] with respect to the inner product (19). This is the reason why they are used as the representation basis in the solutions (23) and (25).

Putting everything together, after applying Helmholtz decomposition, we get the result we want to highlight

$$
\text { if } \begin{aligned}
\omega^{2} & \neq \omega_{n}^{2}, \quad E=E_{0}+e=\frac{1}{i \omega} F_{0}+i \omega \sum_{n=1}^{\infty} \frac{A_{n}}{1-\frac{\omega^{2}}{\omega_{n}^{2}}} e_{n} \\
\text { if } \omega^{2} & =\omega_{n}^{2}, \quad E=E_{0}+e \\
& =\frac{1}{i \omega} F_{0}+i \omega \sum_{\omega_{n}^{2}=\omega^{2}} a_{n} e_{n}+i \omega \sum_{\omega_{n}^{2} \neq \omega^{2}} \frac{A_{n}}{1-\frac{\omega^{2}}{\omega_{n}^{2}}} e_{n}
\end{aligned}
$$

where the frequency dependence in the solution to Maxwell's equations is made explicit [34]. 


\section{REDUCED-BASIS METHOD}

In microwave engineering, we are interested in the frequency behavior in electromagnetic phenomena, where the solution to Maxwell's equations in a specific frequency band is needed. Variational formulation to Maxwell's equations (2) turns into a frequency-parameter variational problem, viz.,

Find $E(\omega) \in \mathcal{H}$ such that

$$
a(E(\omega), v ; \omega)=f(v ; \omega) \quad \forall v \in \mathcal{H} ; \forall \omega \in \mathcal{B}
$$

where $\mathcal{B}=\left[\omega_{\min }, \omega_{\max }\right]$ is the frequency band of interest, and frequency-parameter bilinear and linear forms $a(\cdot, \cdot ; \omega)$ and $f(\cdot ; \omega)$ are already defined in (3) and (4), respectively. This time, all frequency dependence is highlighted. Note that $\mathcal{H}$ is still the infinite dimension admissible function space detailed in (6). This problem can be solved as many times as frequency points are needed by means of the FEM, which results in a rather time-consuming task. However, a better strategy can be proposed and this is where the reduced-basis method comes in place [20], [21].

The key feature upon which the reduced-basis method stands is based on the following assumption. The electromagnetic field does not arbitrarily vary as a function of frequency; in other words, it is not an arbitrary element in $\mathcal{H}$. Instead, it evolves in a simple manifold induced by the frequency parameter $\mathcal{M}_{\omega}=\{E(\omega), \omega \in \mathcal{B}\}$. The assumption here is the claim that $\mathcal{M}_{\omega}$ is a manifold of low dimension, i.e., it can be approximated by a Hilbert space of low dimension $\mathcal{H}_{N}$, which we refer to as reduced-basis space. If it were so, we could convert problem (29) into a more efficient one to solve the variational problem, namely,

$$
\begin{aligned}
\text { Find } E(\omega) & \in \mathcal{H}_{N} \text { such that } \\
a(E(\omega), v ; \omega) & =f(v ; \omega) \quad \forall v \in \mathcal{H}_{N} ; \forall \omega \in \mathcal{B}
\end{aligned}
$$

since the dimension of the reduced-basis space $\mathcal{H}_{N}$ is a small number. The key point here is to provide the reduced-basis space $\mathcal{H}_{N}$ approximating the manifold $\mathcal{M}_{\omega}$, instead of providing an approximation for an arbitrary member in $\mathcal{H}$, such as what FEM proposes, since the field solution $E(\omega)$ in the frequency band of interest does not cover the entire space $\mathcal{H}$.

\section{A. Reduced-Basis Space}

Considering the frequency behavior in the electromagnetic field (28), a compact basis to solve Maxwell's equations in a frequency band of interest $\mathcal{B}$ can be addressed, resulting in an appropriate reduced-basis space. In this section, we focus on the electromagnetic field $E(\omega)$ in the band $\mathcal{B}$ and study its behavior in order to propose a suitable reduced-basis solution to Maxwell's equations.

First of all, as shown in Section II, let us point out that the solution to Maxwell's equations in the whole electromagnetic spectrum requires indeed the knowledge of an infinite number of orthogonal field solutions, $\left\{F_{0}, e_{n} \mid n \in \mathbb{N}\right\}$, which result in a complete solution basis [see (28)]. Recall that $F_{0}$ is the solution to problem (15) and $\left\{e_{n} \mid n \in \mathbb{N}\right\}$ are the eigenfunctions in the eigenproblem (27). As discussed in Section II, all these solutions are orthogonal with respect to the inner product $(\cdot, \cdot)_{\mu, \epsilon}$. Now, our interest is focused on a specific frequency band $\mathcal{B}$ and we can proceed as follows.

1) Either solve for this frequency band $\mathcal{B}$ by using the same complete solution basis for the whole electromagnetic spectrum. This may give rise to rather slowly convergent series, shown in (28).

2) Or find a more compact solution basis for this frequency band $\mathcal{B}$. This can be done by rearranging the field solutions in the basis that are contributing the most to the electromagnetic behavior in $\mathcal{B}$. In contrast to the previous scenario, this will result in a fast convergent electromagnetic-field series expansion.

The second scenario is preferred. For compact representation, we need to identify a solution basis where each vector in the basis is adding the maximum energy to the system among all field solutions $E(\omega)$ in $\mathcal{B}$. In linear algebra terms, we need to add vectors to the solution basis that are the most linearly independent from the previous vectors in the basis, and this should be done among all the field solutions $E(\omega)$ in $\mathcal{B}$, i.e., in the solution manifold $\mathcal{M}_{\omega}$. At first glance, this may seem difficult to do, but let us rearrange (28) in two parts: a regular part in frequency in $\mathcal{B}, F(\omega)$, and a singular part in frequency in $\mathcal{B}, e_{\mathcal{B}}(\omega)$, namely,

$$
\begin{aligned}
E(\omega) & =F(\omega)+e_{\mathcal{B}}(\omega), \quad \omega \in \mathcal{B}=\left[\omega_{\min }, \omega_{\max }\right] \\
F(\omega) & =\frac{1}{i \omega} F_{0}+i \omega \sum_{\omega_{n}^{2} \notin \mathcal{B}_{2}} \frac{A_{n}}{1-\frac{\omega^{2}}{\omega_{n}^{2}}} e_{n} \\
\text { if } \omega^{2} & \neq \omega_{n}^{2}, \quad e_{\mathcal{B}}(\omega)=i \omega \sum_{\omega_{n}^{2} \in \mathcal{B}_{2}} \frac{A_{n}}{1-\frac{\omega^{2}}{\omega_{n}^{2}}} e_{n} \\
\text { if } \omega^{2} & =\omega_{n}^{2}, \quad e_{\mathcal{B}}(\omega) \\
& =i \omega \sum_{\omega_{n}^{2}=\omega^{2}} a_{n} e_{n}+i \omega \sum_{\omega_{n}^{2} \in \mathcal{B}_{2} \backslash\left\{\omega^{2}\right\}} \frac{A_{n}}{1-\frac{\omega^{2}}{\omega_{n}^{2}}} e_{n} .
\end{aligned}
$$

$\mathcal{B}_{2}$ stands for $\left[\omega_{\min }^{2}, \omega_{\max }^{2}\right]$. This rearrangement is also depicted in Fig. 1. It should be noted that the eigenfunctions hit in the frequency band of interest $\left\{e_{n} \mid \omega_{n}^{2} \in \mathcal{B}_{2}\right\}$ are a good choice to be included in the compact solution basis, not only because they are indeed in the manifold $M_{\omega}$, i.e., they are present in the electromagnetic field $E(\omega)$ in $\mathcal{B}$, but also since they form an orthogonal system. As a result, they provide most electromagnetic energy in $\mathcal{B}$. Thus far, the singular part in $(31), e_{\mathcal{B}}(\omega)$, is accurately represented in the solution basis. An additional effort needs to be done to describe the regular part in frequency, $F(\omega)$, in (31).

It should be pointed out that $F(\omega)$ can be represented by the eigenmodes outside the band $\mathcal{B}$ and the curl-free solution $F_{0}$ as described in (31), but this may result in a rather slowly convergent series and an alternative representation basis is sought. However, since the remaining missing part in the solution basis, namely, $F(\omega)$, is a smooth function in frequency, we expect good convergence properties in the reduced-basis method, provided the basis is uplifted with suitable additional vectors. We complete the solution basis by adding field solutions $E(\omega)$ in $\mathcal{B}$ that are the most linearly independent from the previous vectors in the basis. Proceeding in this way ensures that the solution manifold $\mathcal{M}_{\omega}$ is approximated by 

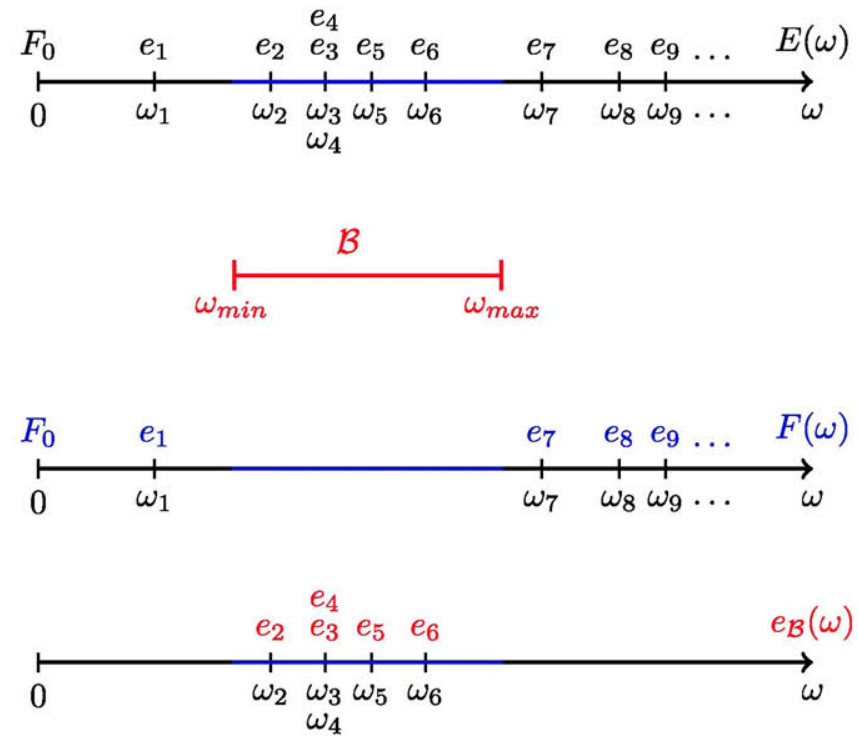

Fig. 1. Electromagnetic field $E(\omega)$ is twofold in the frequency band $\mathcal{B}$ : a regular part in frequency, $F(\omega)$, and a singular part in frequency, $e_{\mathcal{B}}(\omega)$.

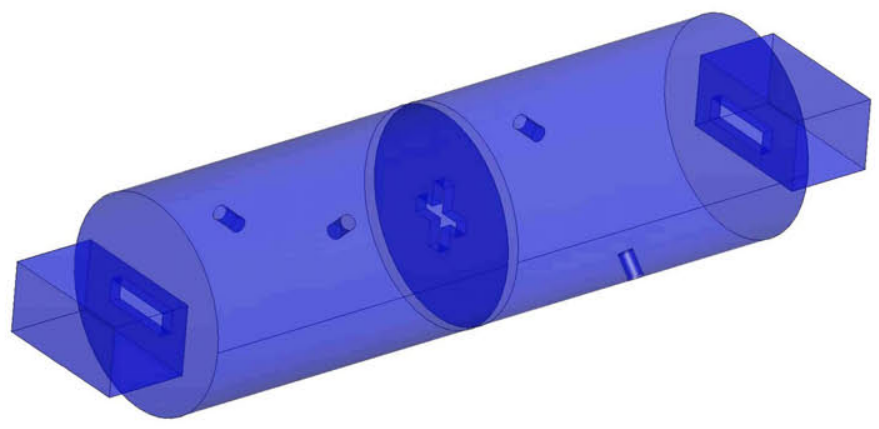

Fig. 2. Dual-mode circular-waveguide filter geometry. Cavity length: $43.87 \mathrm{~mm}$. Radius: $14 \mathrm{~mm}$. Iris thicknesses: $1.5 \mathrm{~mm}$. Slot lengths: $10.05 \mathrm{~mm}$. Slot widths: $3 \mathrm{~mm}$. Arm widths: $2 \mathrm{~mm}$. Horizontal arm length: $7.65 \mathrm{~mm}$. Vertical arm length: $8.75 \mathrm{~mm}$. Tuning screw depth: $3.59 \mathrm{~mm}$. Coupling screw depth: $3.31 \mathrm{~mm}$.

a reduced-basis space with the best solution basis of finite dimension made up of field solution samples.

Let us provide a real-life example where these ideas are considered. Fig. 2 shows a dual-mode circular waveguide filter as well as its geometry dimensions. Two cylindrical cavities are connected through identical slots to the corresponding input and output WR75 rectangular waveguides. In turn, a crossshaped iris connects both cylindrical cavities. A horizontal tuning screw and a tilt coupling screw are included in each cavity. Although two physical cavities are considered, two degenerated modes are excited in each cavity. This gives rise to four electrical cavities reducing the filter size and the weight. As a result, four eigenmodes are expected in the filter operation frequency band $\mathcal{B}$ from 11.5 to $12 \mathrm{GHz}$.

FEM analysis is carried out to study the electromagnetic behavior in this filter. Solving for the eigenmodes in the frequency band $\mathcal{B}$, we find the following eigenresonances: $f_{1}=11.763 \mathrm{GHz}, f_{2}=11.808 \mathrm{GHz}, f_{3}=11.888 \mathrm{GHz}$, and $f_{4}=11.891 \mathrm{GHz}, \omega_{i}=2 \pi f_{i}$, and its corresponding eigenmodes $e_{1}, e_{2}, e_{3}$, and $e_{4}$. These eigenmodes are an orthogonal system with respect to the inner product $(\cdot, \cdot)_{\mu, \epsilon}$ and stand for the singular part in frequency in the electromagnetic field $E(\omega)$ in $\mathcal{B}$, i.e., $e_{\mathcal{B}}(\omega)$. As a result, $\left\{e_{1}, e_{2}\right.$, $\left.e_{3}, e_{4}\right\}$ is the best basis to describe $e_{\mathcal{B}}(\omega)$. However, this basis still needs to be uplifted to accurately describe the solution manifold $\mathcal{M}_{\omega}$, since the regular part behavior in $E(\omega)$ in $\mathcal{B}$, $F(\omega)$, is not considered in this eigenmode basis.

The eigenmode basis $\left\{e_{1}, e_{2}, e_{3}, e_{4}\right\}$ is completed by adding the most linearly independent field solution $E(\omega)$ in $\mathcal{B}$, with respect to the vectors in the basis, one at a time. This maximizes the contribution to the actual electromagnetics in $\mathcal{B}$, i.e., the electromagnetic energy, that is added to the representation basis for each new vector. Once again, let us point out that, since the missing electromagnetic contribution to the eigenmode basis, $F(\omega)$, is a smooth function in frequency in $\mathcal{B}$, it is expected that, most of the electromagnetic energy in the solution manifold $\mathcal{M}_{\omega}$ be represented by a small number of additional vectors. This supports the application of the reduced-basis method to solve Maxwell's equations, where an appropriate reduced-basis space can be identified.

In order to measure the linear independence of vector $E$ with respect to the basis $B_{n}=\left\{e_{1}, \ldots, e_{n}\right\}$, Algorithm 1 is proposed.

$\overline{\text { Algorithm 1 Linear Independence Measure }}$
\[ G=E /\|E\| \]

2) Orthonormalize $B_{n}=\left\{e_{1}, \ldots, e_{n}\right\}$ by Gram-Schmidt.

$$
D_{n}=\left\{g_{1}, \ldots, g_{n}\right\}
$$

3) Project $G$ onto the orthonormal basis $D_{n}$.

$$
g=\sum_{k=1}^{n}\left(G, g_{k}\right) g_{k}
$$

4) Represent $G$ in terms of its projection onto $D_{n}, g$, and its orthogonal complement, $g_{\perp}$.

$$
G=g+g_{\perp}
$$

5) Compute the norm of $g_{\perp}$.

$$
\mu_{B_{n}}=\left\|g_{\perp}\right\|=\|G-g\|
$$

In Algorithm 1, the figure of merit $\mu_{B_{n}}$ is a measure of how linearly independent $E$ is with respect to the basis $B_{n}$. Indeed, $\mu_{B_{n}}$ takes values in the interval $[0,1]$, where $\mu_{B_{n}}=0$ indicates that $E$ is linearly dependent on $B_{n}$, and progressively increases up to $\mu_{B_{n}}=1$, which shows that $E$ is the most linearly independent from $B_{n}$, i.e., orthogonal to the basis $B_{n}$.

Let us apply this linear independence measure to the field solutions $E(\omega)$, computed by FEM, in the $11.5-12-\mathrm{GHz}$ band in the dual-mode filter example in Fig. 2. The inner product $(\cdot, \cdot)_{\mu, \epsilon}$ is considered in Algorithm 1. We start from the eigenbasis $B_{4}=\left\{e_{1}, e_{2}, e_{3}, e_{4}\right\}$ described earlier. Fig. 3(a) details how linearly independent the field solution $E(\omega)$ is 


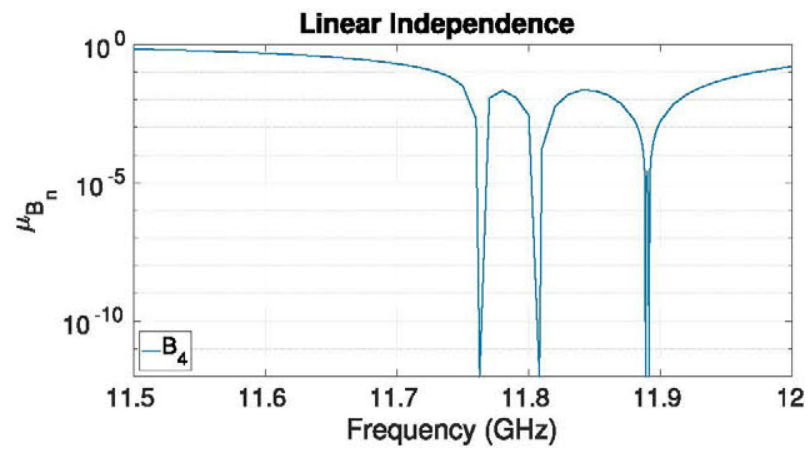

(a)

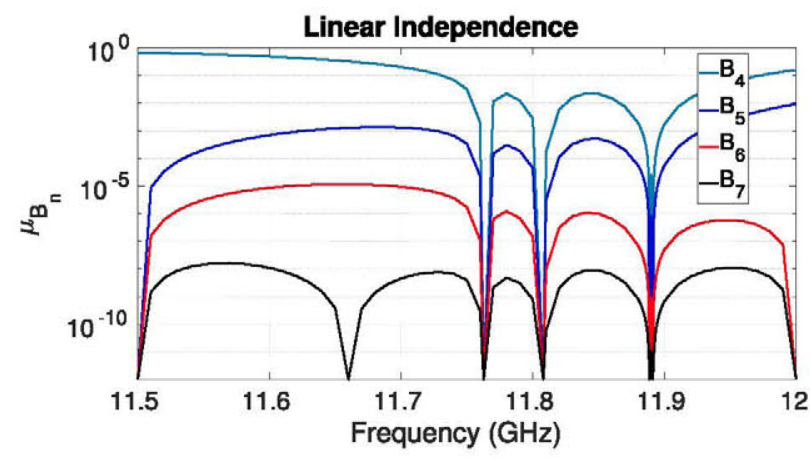

(b)

Fig. 3. Linear independence results in the field solutions $E(\omega)$ for the dual-mode circular waveguide filter. (a) Independence with respect to the eigenbasis $B_{4}$. (b) Independence with respect to eigenbasis $B_{4}$ and progressively enriched solution basis $B_{5}, B_{6}$, and $B_{7}$.

with respect to the eigenbasis $B_{4}$. It should be noted that $E(\omega)$ is linearly dependent on this eigenbasis $B_{4}$ exactly at the eigenresonances, i.e., $f_{1}=11.763 \mathrm{GHz}, f_{2}=11.808 \mathrm{GHz}$, $f_{3}=11.888 \mathrm{GHz}$, and $f_{4}=11.891 \mathrm{GHz}$. It is also shown in Fig. 3(a) that certain linear dependence is kept in some neighborhood at the eigenresonances, since the field pattern around eigenresonance $\omega_{n}$ is dominated by the corresponding eigenmode $e_{n}$. This can also be seen in (28). The further away from eigenresonances we are, the more linearly independent the electromagnetic field $E(\omega)$ becomes, and eventually, it will become orthogonal $\left(\mu_{B_{n}}=1\right)$ when we hit the next out-ofband eigenresonance, outside $\mathcal{B}$.

Fig. 3(b) details the linear independence of the electromagnetic field $E(\omega)$ with respect to the enriched solution basis $B_{5}, B_{6}$, and $B_{7}$, when each of these solution bases is uplifted with the most linearly independent field solution in $\mathcal{B}$, one at a time, namely,

$$
\begin{aligned}
& w_{n+1}=\arg \max _{\omega \in \mathcal{B}} \mu_{B_{n}}(E(\omega)) \\
& B_{n+1}=B_{n} \cup\left\{E\left(w_{n+1}\right)\right\} .
\end{aligned}
$$

It should be pointed out that $w$ notation is used now instead of $\omega$ in order to indicate that these field solutions $E\left(w_{n+1}\right)$ are no longer eigenmodes, and as a result, these frequencies $w_{n+1}$ are not eigenresonances any longer. These results suggest that, following this selection criterion, most of the electromagnetic behavior in the frequency band of interest $\mathcal{B}$ can be appropriately represented by a solution basis of



(a)

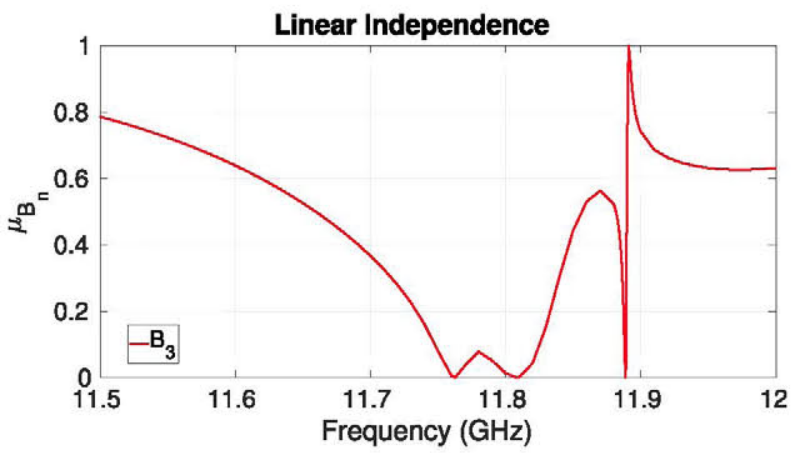

(b)

Fig. 4. Linear independence results in the field solutions $E(\omega)$ for the dual-mode circular-waveguide filter. (a) Independence with respect to the eigenmodes in the basis $B_{1}, B_{2}, B_{3}$, and $B_{4}$. (b) Independence with respect to the eigenmodes $e_{1}, e_{2}$, and $e_{3}$ in the basis $B_{3}$.

finite dimension. In other words, the solution manifold $\mathcal{M}_{\omega}$ can indeed be conveniently approximated by a Hilbert space of finite dimension $\mathcal{H}_{N}$, namely, a reduced-basis space.

In order to show further insight, the linear independence of the electromagnetic field $E(\omega)$ with respect to the eigenmodes used in the eigenbasis $B_{4}=\left\{e_{1}, e_{2}, e_{3}, e_{4}\right\}$ is addressed in Fig. 4. It is noted that $E(\omega)$ is linearly dependent on the eigenresonances in $B_{n}\left(\mu_{B_{n}}=0\right)$ and becomes orthogonal $\left(\mu_{B_{n}}=1\right)$ at the eigenresonances not included in the basis $B_{n}$. No matter how close these eigenresonances are, the transition for an electromagnetic field $E(\omega)$ to be included in the representation basis $B_{n}$, i.e., $\mu_{B_{n}}=0$, or to become completely orthogonal, $\mu_{B_{n}}=1$, can be very fast. This is detailed in Fig. 4(b) and it is due to the fact that eigenresonances $f_{3}=11.888 \mathrm{GHz}$ and $f_{4}=11.891 \mathrm{GHz}$ are very close to each other. This is showing how important is to include all eigenmodes hit in the frequency band of analysis in the reduced-basis space.

Finally, Table I details the field solutions added to the reduced-basis following selection criterion (32), as well as its linear independence with respect to the previously built basis and the frequency samples chosen.

\section{B. Reduced-Basis Approximation}

It has been detailed that the reduced-basis method can be applied to solve Maxwell's equations in a frequency band of interest $\mathcal{B}$ by means of the identification of a reduced-basis 
TABLE I

FIELD SOLUTIONS FOUND BY CRITERION (32) FOR REDUCED-BASIS APPROXIMATION IN THE DUAL-MODE CIRCUL AR-WAVEGUIDE FILTER

\begin{tabular}{c|c|c|c}
\hline Frequency (GHz) & Field & Basis & $\mu_{B_{n}}\left(E_{n+1}\right)$ \\
\hline \hline 11.763 & $e_{1}$ & - & - \\
11.808 & $e_{2}$ & $B_{1}$ & 1. \\
11.888 & $e_{3}$ & $B_{2}$ & 1. \\
11.891 & $e_{4}$ & $B_{3}$ & 1. \\
11.500 & $E_{5}$ & $B_{4}$ & 0.676 \\
12.000 & $E_{6}$ & $B_{5}$ & $9.376 \cdot 10^{-3}$ \\
11.660 & $E_{7}$ & $B_{6}$ & $1.160 \cdot 10^{-5}$ \\
11.570 & $E_{8}$ & $B_{7}$ & $1.619 \cdot 10^{-8}$ \\
11.960 & $E_{9}$ & $B_{8}$ & $4.571 \cdot 10^{-10}$ \\
\hline
\end{tabular}

space. This reduced-basis space is set up by the eigenmodes hit in the frequency band of analysis $\left\{e_{1}, \ldots, e_{n}\right\}$ and completed by electromagnetic fields $E(\omega)$ properly selected in the frequency band of analysis $\mathcal{B}$. It should be noted that the selection criterion (32) to complete the eigenbasis is hard to follow in practice, but an alternative, easy to compute selection criterion can be addressed. We will comment on this in further detail in Section III-C.

Assuming that the following reduced-basis space $\mathcal{H}_{N}$ of dimension $N$ is considered:

$$
\mathcal{H}_{N}=\operatorname{span}\left\{\left\{e_{1}, \ldots, e_{n}\right\} \cup\left\{E\left(w_{n+1}\right), \ldots, E\left(w_{N}\right)\right\}\right\}
$$

where frequencies $w_{n+1}, \ldots, w_{N}$ are properly chosen within the frequency band $\mathcal{B}$, let us carry out the reduced-basis approximation to the frequency-parameter weak formulation for Maxwell's equations (29), viz.,

$$
\begin{aligned}
\text { Find } \tilde{E}(\omega) & \in \mathcal{H}_{N} \text { such that } \\
a(\tilde{E}(\omega), v ; \omega) & =f(v ; \omega) \quad \forall v \in \mathcal{H}_{N} ; \forall \omega \in \mathcal{B} .
\end{aligned}
$$

It should be noted that $\tilde{E}(\omega)$ is an approximation to the actual electromagnetic field $E(\omega)$ in $\mathcal{B}$; the better job we do in completing the reduced-basis space to span the solution manifold $\mathcal{M}_{\omega}$, the better the reduced-basis approximation will be, that is

$$
E(\omega) \simeq \tilde{E}(\omega)=\sum_{k=1}^{n} \alpha_{k}(\omega) e_{k}+\sum_{k=n+1}^{N} \beta_{k}(\omega) E\left(w_{k}\right)
$$

where $\alpha_{k}(\omega)$ and $\beta_{k}(\omega)$ can straightforwardly be found for any frequency by solving the reduced-order variational problem (34). In addition, the frequency-parameter bilinear and linear forms $a(\cdot, \cdot ; \omega)$ and $f(\cdot ; \omega)$ exhibit an affine frequency-parameter dependence, namely,

$$
\begin{aligned}
a(u, v ; \omega) & =\left(\frac{1}{\mu} \nabla \times u, \nabla \times \bar{v}\right)_{L^{2}(\Omega)}-\omega^{2}(\varepsilon u, \bar{v})_{L^{2}(\Omega)} \\
f(v ; \omega) & =i \omega\langle J, n \times v \times n\rangle .
\end{aligned}
$$

The frequency-independent products among all the elements in the reduced-basis space (33) can be computed once and stored in memory. This improves the assembly process, where all time-consuming operations are carried out only once offline and stored in memory, and every time a new frequency solution $\tilde{E}(\omega)$ is needed online, this solution can be found involving very few operations. This is known as the offine-online decomposition and it is used to speed up the reduced-basis approximation, providing a very fast parameter sweep in the solution to the problem [20]-[22].

\section{Reliability in the Reduced-Basis Approximation}

Not only is a fast frequency sweep field solution desired, but also a reliable one. In order to certify the reliability of the reduced-basis approximation, special attention should be placed in completing the reduced-basis space. As has already been mentioned, the selection criterion (32) to complete the eigenbasis is not of practical use, since it requires the actual computation of the field solutions in the frequency band of analysis, which is actually what we are aimed for within the reduced-basis method. However, an alternative strategy can be proposed to complete the reduced-basis space in a straightforward way. This is based on the fast evaluation of the residual error in the original problem with respect to the actual field solution $E(\omega)$ for the reduced-basis approximation solution $\tilde{E}(\omega)$, taking advantage of the affine parameter dependence and the field solution structure in frequency. The residual error is given as follows:

$$
\begin{aligned}
r(\tilde{E}(\omega), v ; \omega) & =a(E(\omega)-\tilde{E}(\omega), v ; \omega) \\
& =f(v ; \omega)-a(\tilde{E}(\omega), v ; \omega) \quad \forall v \in \mathcal{H} .
\end{aligned}
$$

It should be pointed out that the residual error $r(\tilde{E}(\omega), \cdot, \omega)$ is a linear functional in $\mathcal{H}$, and therefore an element in its dual space $\mathcal{H}^{\prime}$. We quantify this residual by its norm

$$
\left.\|r(\tilde{E}(\omega), \cdot ; \omega)\|\right|_{\mathcal{H}^{\prime}}=\sup _{v \in \mathcal{H}} \frac{|r(\tilde{E}(\omega), v ; \omega)|}{\|v\|_{\mathcal{H}}} .
$$

We can apply the Riesz representation theorem in order to find an isometric representative, namely, Riesz representative $\tilde{\epsilon}(\omega)$, to the residual $r(\tilde{E}(\omega), \cdot, \omega)$, that is

$$
\begin{aligned}
(\tilde{\epsilon}(\omega), v)_{\mu, \varepsilon} & =r(\tilde{E}(\omega), v ; \omega) \quad \forall v \in \mathcal{H} \\
\|\tilde{\epsilon}(\omega)\|_{\mathcal{H}} & =\|r(\tilde{E}(\omega), \cdot ; \omega)\|_{\mathcal{H}^{\prime}} .
\end{aligned}
$$

Since the residual error (37) exhibits an affine frequency-parameter dependence, we can carry out an offline-online decomposition in order to quantify this residual error in the whole frequency band of analysis $\mathcal{B}$ in an efficient way [22].

Let us define the following efficient alternative criterion to (32) to complete the eigenbasis in the reduced-basis space, namely:

$$
\begin{aligned}
& w_{n+1}=\arg \max _{\omega \in \mathcal{B}} v_{B_{n}}(\tilde{E}(\omega)) \\
& B_{n+1}=B_{n} \cup\left\{E\left(w_{n+1}\right)\right\}
\end{aligned}
$$

where $v_{B_{n}}(\tilde{E}(\omega))$ is the normalized residual error norm of the reduced-basis approximation $\tilde{E}(\omega)$ in $B_{n}$, that is,

$$
v_{B_{n}}(\tilde{E}(\omega))=\frac{\|r(\tilde{E}(\omega), \cdot ; \omega)\|_{\mathcal{H}^{\prime}}}{\|f(\cdot ; \omega)\|_{\mathcal{H}^{\prime}}}
$$


Again, $w$ notation is used instead of $\omega$ in order to indicate that these field solutions $E\left(w_{n+1}\right)$ are no longer eigenmodes and, as a result, these frequencies $w_{n+1}$ are not eigenresonances. Following the selection criterion (40), the reducedbasis space is enhanced by field solutions $E\left(w_{n+1}\right)$ that are contributing the most to the residual error, one at a time. This is known as greedy algorithm. It should be noted that this residual can be computed very fast in the frequency band of interest [22], which makes this selection criterion of practical use. On the other hand, the residual error norm itself is not the best indicator to monitor when the solution manifold $\mathcal{M}_{\omega}$ is properly described by the reduced-basis space and when the greedy algorithm to increase the reduced-basis space should stop. It is the electric field itself the quantity that should be monitored in this regard. In this sense, the measure of the linear independence $\mu_{B_{n}}\left(E\left(w_{n+1}\right)\right)$ at this properly selected new frequency $w_{n+1}$ is preferred as the stopping criterion, since this quantity actually certifies how much new information is added to the reduced-basis space in order to accurately approximate the solution manifold $\mathcal{M}_{\omega}$. A complete algorithm to carry out a reliable reduced-basis approximation resulting in a fully automatic model-order reduction will be described at the end of this section.

There is still an issue that deserves further discussion. The residual error due to an eigenmode approximation in Maxwell's equations has a specific frequency behavior in the whole electromagnetic spectrum, which will be further detailed. This residual error frequency behavior should be considered in the greedy algorithm (40). Due to this fact, it cannot be used at the initial stage of the algorithm. To explain it, let us go through the details. Let us solve for the reduced-basis approximation $\tilde{E}(\omega)$ within the reduced-basis space $\mathcal{H}_{n}$ spanned by the eigenmodes $\left\{e_{1}, \ldots, e_{n}\right\}$ with the corresponding eigenresonances $\left\{\omega_{1}, \ldots, \omega_{n}\right\}$, i.e., $\mathcal{H}_{n}=$ $\operatorname{span}\left\{e_{1}, \ldots, e_{n}\right\}$, namely,

$$
\text { Find } \tilde{E}(\omega) \in \mathcal{H}_{n} \text { such that }
$$

$$
a(\tilde{E}(\omega), v ; \omega)=f(v ; \omega) \quad \forall v \in \mathcal{H}_{n}, \quad \forall \omega \in \mathcal{B}
$$

resulting in

$$
\tilde{E}(\omega)=\sum_{k=1}^{n} \alpha_{k}(\omega) e_{k}
$$

where $\alpha_{k}(\omega)$ are obtained by solving the reduced-order variational problem (42) frequency by frequency. In this framework, the frequency behavior in the reduced-basis approximation $\tilde{E}(\omega)$ is known a priori. Indeed, variational problem (42) reduces to

Find $\tilde{E}(\omega) \in \mathcal{H}_{n}$ such that

$$
\begin{array}{r}
\left(\frac{1}{\mu} \nabla \times \tilde{E}(\omega), \nabla \times \bar{v}\right)_{L^{2}(\Omega)}-\omega^{2}(\varepsilon \tilde{E}(\omega), \bar{v})_{L^{2}(\Omega)} \\
=i \omega\langle j, n \times v \times n\rangle \quad \forall v \in \mathcal{H}_{n} ; \quad \forall \omega \in \mathcal{B} .
\end{array}
$$

It should be pointed out that the linear functional $f(v ; \omega)=$ $i \omega\langle J, n \times v \times n\rangle$ reduces to $f(v ; \omega)=i \omega\langle j, n \times v \times n\rangle, \forall v \in$ $\mathcal{H}_{n}$, where the splitting (12) has been considered, i.e., $J=$ $J_{0}+j, J_{0} \in \mathcal{U}, j \in \mathcal{W}$. This is the case due to the orthogonality $\left\langle J_{0}, n \times \bar{v} \times n\right\rangle=0, \forall 0 \in \mathcal{H}_{n}$, as it will become clear in the following. Recall that the basis in $\mathcal{H}_{n}$ is made up of real-valued eigenmodes, and in particular, $\bar{e}_{k}$ can be replaced by $e_{k}$. This orthogonality holds true not only for the eigenmodes in $\mathcal{H}_{n}$, but also for all the eigenmodes in $\mathcal{V}$, as it will be detailed next. Recall that the Helmholtz decomposition (9) is used, i.e., $E=E_{0}+e, E_{0} \in \mathcal{H}(\operatorname{curl} 0, \Omega), e \in \mathcal{V}$. In order to show this orthogonality result, let us proceed by testing (14) in $\mathcal{V}$ introducing the solution for $e$ (18). This yields

$$
-\omega^{2}\left(\varepsilon E_{0}, v\right)_{L^{2}(\Omega)}=i \omega\left\langle J_{0}, n \times \bar{v} \times n\right\rangle \quad \forall v \in \mathcal{V}
$$

where $\varepsilon$ is a real-valued function, since our medium is assumed

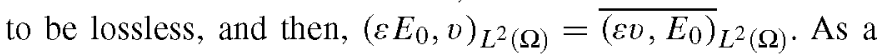
result of the definition in the Helmholtz decomposition (10b), $\left(\varepsilon v, E_{0}\right)_{L^{2}(\Omega)}=0, \forall v \in \mathcal{V}$ giving rise to

$$
\left\langle J_{0}, n \times \bar{v} \times n\right\rangle=0 \quad \forall v \in \mathcal{V} .
$$

Now, we continue with the solution of the reduced-basis problem (44). Following an analogous reasoning as in Section II, the frequency behavior of the reduced basis approximation $\tilde{E}(\omega)$ reads, either:

$$
\tilde{E}(\omega)=i \omega \sum_{k=1}^{n} \frac{A_{k}}{1-\frac{\omega^{2}}{\omega_{k}^{2}}} e_{k} \text { if } \omega^{2} \neq \omega_{k}^{2}
$$

where

$$
A_{k}=\left\langle j, n \times \bar{e}_{k} \times n\right\rangle
$$

or

$$
\tilde{E}(\omega)=i \omega \sum_{\omega_{k}^{2}=\omega^{2}} a_{k} e_{k}+i \omega \sum_{\omega_{k}^{2} \in \in \mathcal{B}_{2} \backslash\left\{\omega^{2}\right\}} \frac{A_{k}}{1-\frac{\omega^{2}}{\omega_{k}^{2}}} e_{k} \text { if } \omega^{2}=\omega_{k}^{2}
$$

where

$$
\left\langle j, n \times \bar{e}_{k} \times n\right\rangle=0, \quad \text { for } \omega_{k}^{2}=\omega^{2} .
$$

Here, $a_{k}$ are arbitrary constants, since the solution is not unique whenever $\omega^{2}=\omega_{k}^{2}$, and $A_{k}$ are defined as in (48). Recall that $\mathcal{B}_{2}$ stands for $\left[\omega_{\min }^{2}, \omega_{\max }^{2}\right]$.

The fact we would like to point out is that this reduced-basis approximation (47) and (49) gives rise to the following residual error:

$$
\begin{aligned}
r(\tilde{E}(\omega), v ; \omega) & =f(v ; \omega)-a(\tilde{E}(\omega), v ; \omega) \\
& =i \omega\langle J, n \times v \times n\rangle-i \omega\left\langle J, n \times \pi_{\mathcal{H}_{n}}(v) \times n\right\rangle \\
& =i \omega\left\langle J, n \times \pi_{\mathcal{G}}(v) \times n\right\rangle \quad \forall v \in \mathcal{H}
\end{aligned}
$$

where the orthogonal decomposition $\mathcal{H}=\mathcal{G} \oplus \mathcal{H}_{n}$ is considered and $\pi_{\mathcal{F}}(\cdot)$ stands for the projection operator on $\mathcal{F}$. It should be noted that $a(\tilde{E}(\omega), v ; \omega)=i \omega$ $\left\langle j, n \times \pi_{\mathcal{H}_{n}}(v) \times n\right\rangle$ for all $v \in \mathcal{H}$, but since $\left\langle J_{0}, n \times \pi_{\mathcal{H}_{n}}(v) \times\right.$ $n\rangle=0$ for all $v \in \mathcal{H}$ [see (46)], we can add $J_{0}$ recovering the original current excitation $J=J_{0}+j$. The result obtained in (51) brings to light that all frequency field solutions $\tilde{E}(\omega)$ spanned by the eigenbasis in the reduced-basis approximation, no matter the frequency $\omega$, are contributing in the same amount to the residual error, apart from a $i \omega$ factor. This residual error, when normalized by the norm of the linear functional $f(\cdot ; \omega)$ which also exhibits the same $i \omega$ factor, results in a 


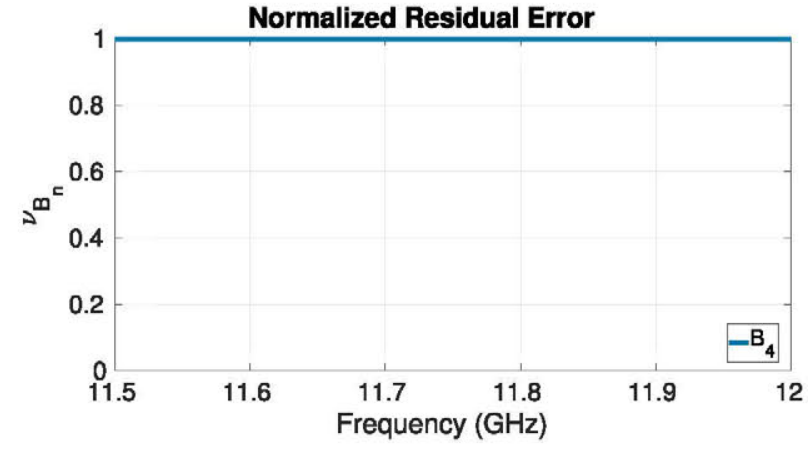

Fig. 5. Normalized residual error in the reduced-basis approximation when only the eigenbasis $B_{4}$ spans the reduced-basis space in the dual-mode filter example. A constant behavior in frequency is observed.

constant value regardless of the frequency. This is depicted in Fig. 5 for the dual-mode filter example in Fig. 2. This particular frequency behavior in the residual error revealed by (51) indicates that the selection criterion (40), based on the normalized residual error, is not of much help at the very first step in completing the reduced-basis space starting from the eigenbasis $B_{n}$, since we get that any frequency field solution in the reduced-basis approximation is contributing in the same amount to the residual. Therefore, once we have included the $n$ eigenmodes in the band, we cannot identify which frequency field solution $E\left(w_{n+1}\right)$ will be contributing the most to enhance the reduced-basis space based on the residual at this initial step (see Fig. 5). A different selection criterion for this special situation when we want to uplift the eigenbasis is needed and, again, we go back to the linear independence reasoning considering the frequency structure in the electromagnetic field (28). Based on this frequency behavior, the further away we are from the eigenresonances $\left\{\omega_{1}, \ldots, \omega_{n}\right\}$ in the frequency band of interest $\mathcal{B}$, the more linearly independent information we find in the electromagnetic field $E(\omega)$, and eventually, we will find the most linear independence, i.e., an orthogonal field, when we hit the next. eigenresonance out of the band of interest. This is shown in Fig. 4 for the dual-mode filter example in Fig. 2. As a result, a good field solution $E\left(w_{n+1}\right)$ to uplift the eigenbasis will be at the end points in the frequency band of interest $\mathcal{B}=\left[\omega_{\min }, \omega_{\max }\right]$. Choosing the best end point requires the knowledge of the next out-of-band eigenresonance. However, picking the end point, either $\omega_{\min }$ or $\omega_{\max }$, that is further away from the in-band eigenresonances $\left\{\omega_{1}, \ldots, \omega_{n}\right\}$ is a good choice, although sometimes it is not the optimum.

Putting everything together, we are able to sum up and carry out an automatic and reliable model-order reduction for fast frequency sweep in microwave devices. Algorithm 2 to build up a compact and reliable reduced-basis approximation is proposed.

It has to be highlighted that the reduced basis that we propose and practical procedure for its identification are remarkably different to the one traditionally used in the reduced-basis method [20], [21]. In particular, we deliberately include eigenmodes in the reduced-basis, which are typically avoided in the reduced-basis method. In addition, the stopping

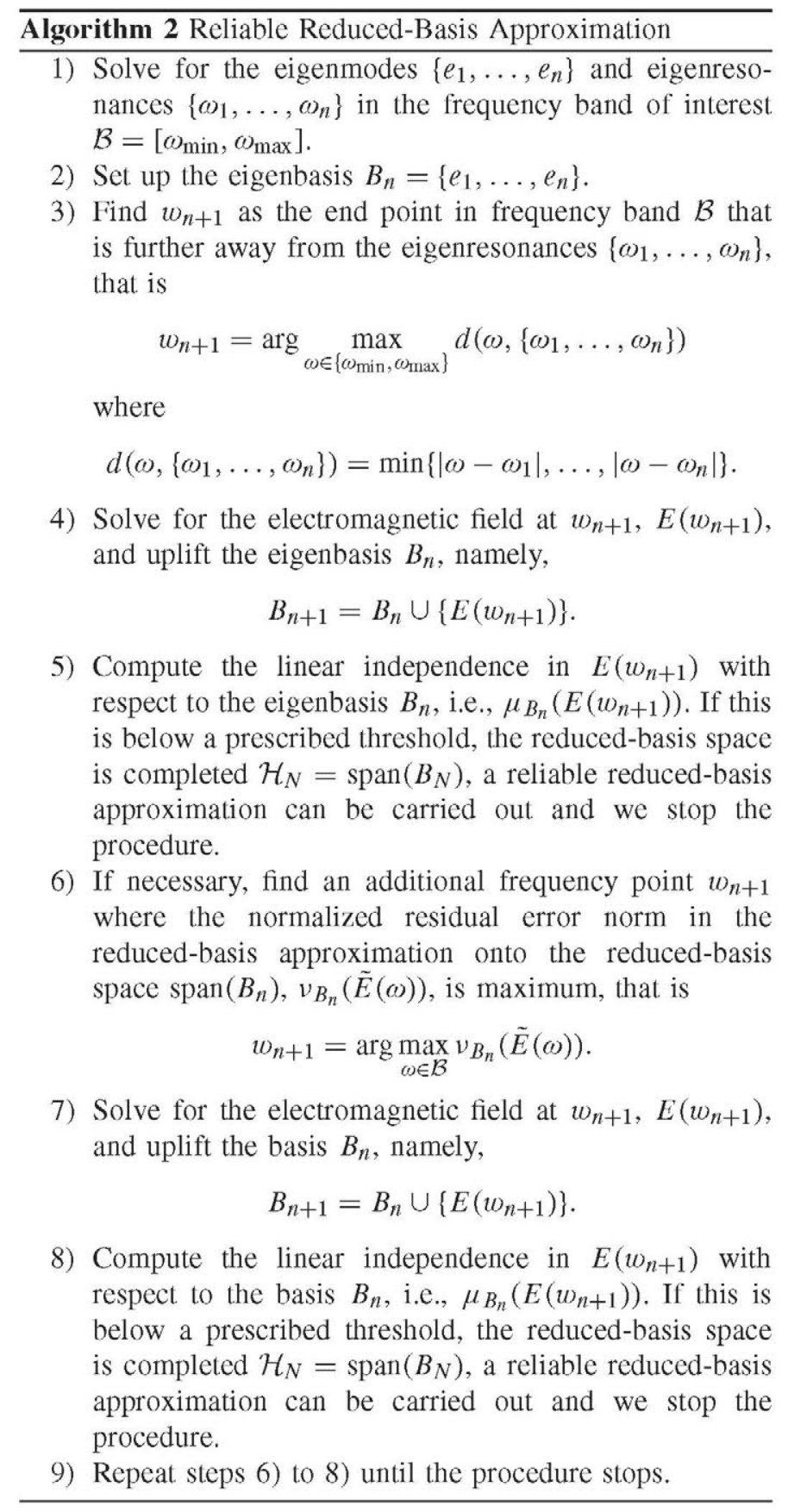

criterion used in this paper is based on linear independence results and differs from the ones suggested in [22]-[25].

\section{NUMERICAL RESULTS}

In this section, we apply the proposed reduced-basis method to different challenging structures, namely, a dualmode circular waveguide filter, a rectangular waveguide filter, a waveguide diplexer, and a Bethe hole waveguide coupler. The capabilities and reliability of the proposed procedure will be apparent throughout these examples. All computations were carried out on a workstation with $3.00-\mathrm{GHz}$ Intel Xeon ES-2687W v4 processor and 64-GB RAM. FEM simulations were carried out with the code described in [35]. 


\section{A. Dual-Mode Circular-Waveguide Filter}

This dual-mode filter has already been described in Fig. 2 and its features have been studied to detail the possibilities in the proposed approach. In this section, we carry out the fast frequency sweep in the 11.5-12-GHz band through the reliable reduced-basis approximation described in Algorithm 2, and show the numerical results obtained in comparison with FEM analysis. As it was already detailed in Section III-A for this example, solving the eigenmodes by means of FEM analysis in the 11.5-12-GHz band, we find the following eigenresonances: $f_{1}=11.763 \mathrm{GHz}, f_{2}=11.808 \mathrm{GHz}, f_{3}=11.888 \mathrm{GHz}$, and $f_{4}=11.891 \mathrm{GHz}$. So far, the eigenbasis made up of these eigenmodes $B_{4}=\left\{e_{1}, \ldots, e_{4}\right\}$ is found. Now, we proceed as detailed in Algorithm 2 finding the end frequency in the band of analysis $\mathcal{B}$ that is further away from the eigenresonances found in $\mathcal{B}$. This is done in an attempt to find the most linearly independent field solution with respect to eigenmodes in the eigenbasis $B_{4}=\left\{e_{1}, \ldots, e_{4}\right\}$. We solve for the electromagnetic field at this frequency $f_{5}=11.5 \mathrm{GHz}$, namely, $E_{5}$, via FEM analysis. Next, we measure how linearly independent this field solution $E_{5}$ is with respect to the previous basis $B_{4}$ computing $\mu_{B_{4}}\left(E_{5}\right)$ detailed in Algorithm 1 . If this value $\mu_{B_{4}}\left(E_{5}\right)$ is low enough, the enhanced basis $B_{5}=B_{4} \cup\left\{E_{5}\right\}$ should be considered as a good basis to span the field solution in the frequency band $\mathcal{B}$ and can be used with confidence for reduced-basis approximation. On the contrary, if this value $\mu_{B_{4}}\left(E_{5}\right)$ is not small enough, we cannot be certain about the reliability of the reduced-basis approximation carried out within the reduced-basis space spanned by this new basis $B_{5}$ in the whole frequency band $\mathcal{B}$, and as a result, it is a good practice to increase the size of the basis by additional field solutions in the band $\mathcal{B}$. Following Algorithm 2, we look for a new field solution $E_{6}$ in the frequency band of interest to be added to the basis $B_{5}$ by finding the frequency $f_{6}$ that maximizes the normalized residual error in the reduced-basis approximation within the reduced-basis space spanned by this basis $B_{5}$, namely, $v_{B_{5}}(\tilde{E}(f))$, which is depicted in Fig. 6(a). This is done in an attempt to find out the most linearly independent field solution, this time guided by the residual error, which can straightforwardly be computed in the whole frequency band $\mathcal{B}$. Then, we carry out an FEM analysis to solve for the electromagnetic field at this frequency $f_{6}$, namely, $E_{6}$. It should be noted that $f_{6}=12 \mathrm{GHz}$, as detailed in Fig. 6(a). We measure how linearly independent this new field solution $E_{6}$ is with respect to the previous basis $B_{5}$, i.e., we compute $\mu_{B_{5}}\left(E_{6}\right)$ as described in Algorithm 1. If this value $\mu_{B_{5}}\left(E_{6}\right)$ is low enough, we should be confident that the uplifted basis $B_{6}=B_{5} \cup\left\{E_{6}\right\}$ spans a good reduced-basis space to carry out a reliable reduced-basis approximation in the whole frequency band $\mathcal{B}$. On the contrary, if this value is not small enough, we had better increase the size of the actual basis $B_{6}$ in order to certify that the reduced-basis approximation carried out is accurate enough. As detailed in Algorithm 2, we repeat these last steps, solving for the residual error in the reduced-basis approximation until we are certain that the reduced-basis space built up is accurate enough to describe the solution manifold $\mathcal{M}_{\omega}$.

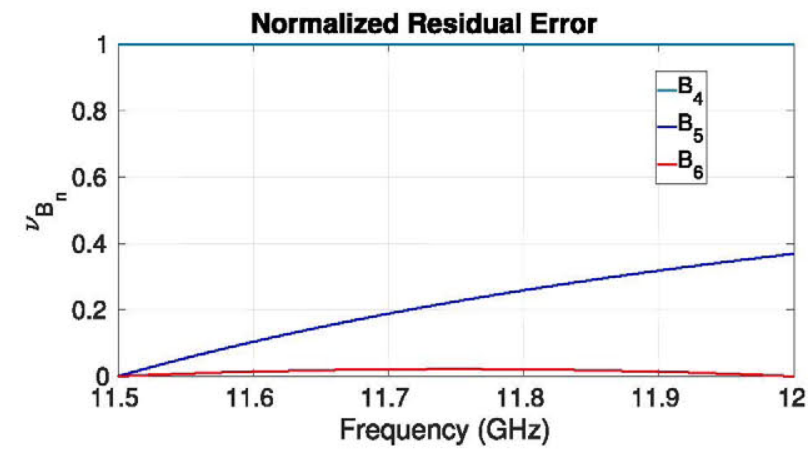

(a)

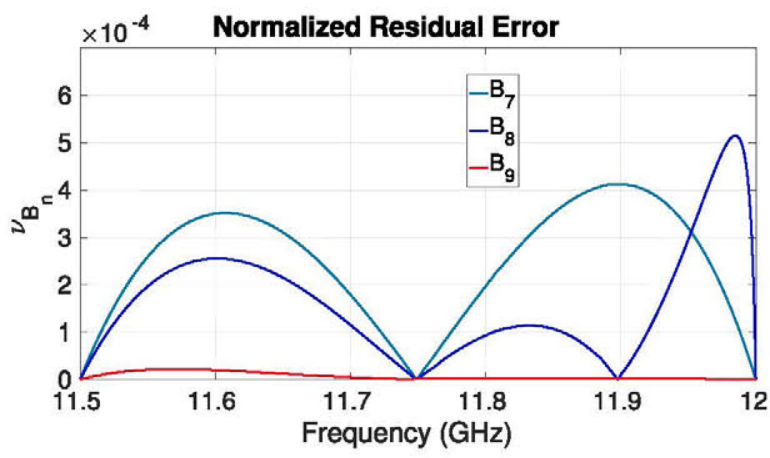

(b)

Fig. 6. Normalized residual error frequency behavior in the reduced-basis approximation as the reduced-basis space is uplifted following the approach detailed in Algorithm 2 in the dual-mode filter example. (a) Normalized residual error in the reduced-basis space spanned by the eigenbasis $B_{4}$ and bases $B_{5}$ and $B_{6}$. A large drop is observed as soon as the eigenbasis $B_{4}$ is complemented. (b) Normalized residual error in the reduced-basis space spanned by bases $B_{7}, B_{8}$, and $B_{9}$.

TABLE II

LINEAR INDEPENDENCE IN THE FIELD SOLUTIONS IN THE DUAL-Mode CirculaR-WAVEguide Filter

\begin{tabular}{c|c|c|c}
\hline Frequency (GHz) & Field & Basis & $\mu_{B_{n}}\left(E_{n+1}\right)$ \\
\hline \hline 11.763 & $e_{1}$ & - & - \\
11.808 & $e_{2}$ & $B_{1}$ & 1. \\
11.888 & $e_{3}$ & $B_{2}$ & 1. \\
11.891 & $e_{4}$ & $B_{3}$ & 1. \\
11.500 & $E_{5}$ & $B_{4}$ & 0.676 \\
12.000 & $E_{6}$ & $B_{5}$ & $9.376 \cdot 10^{-3}$ \\
11.749 & $E_{7}$ & $B_{6}$ & $1.913 \cdot 10^{-6}$ \\
11.898 & $E_{8}$ & $B_{7}$ & $1.530 \cdot 10^{-10}$ \\
11.985 & $E_{9}$ & $B_{8}$ & $5.488 \cdot 10^{-12}$ \\
11.571 & $E_{10}$ & $B_{9}$ & $8.690 \cdot 10^{-11}$ \\
\hline
\end{tabular}

Fig. 6 shows the normalized residual error behavior as we keep on adding field solutions uplifting the reduced-basis space to improve the reduced-basis approximation, as described in Algorithm 2. The linear independence for each of these field solutions with respect to the previously built basis, as well as the frequency samples selected, is detailed in Table II. It should be pointed out that the greedy algorithm based on the residual error (40) does not guarantee that we are choosing the most linearly independent field solutions to 


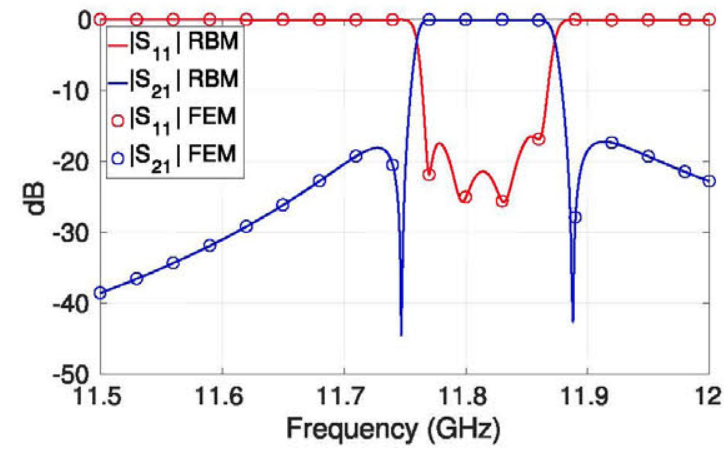

Fig. 7. Dual-mode circular-waveguide filter ROM results are compared with the full-wave FEM scattering parameter response.

TABLE III

POD Singular-VALUE COMPARISON IN THE DUAL-MODE Circular-Waveguide Filter

\begin{tabular}{c|c|c|c}
\hline Field & $\mu_{B_{n}}\left(E_{n+1}\right)$ & Singular vector & Singular value \\
\hline \hline$e_{1}$ & - & $v_{1}$ & 1 \\
$e_{2}$ & 1. & $v_{2}$ & 0.0116 \\
$e_{3}$ & 1. & $v_{3}$ & $3.252 \cdot 10^{-3}$ \\
$e_{4}$ & 1. & $v_{4}$ & $7.536 \cdot 10^{-4}$ \\
$E_{5}$ & 0.676 & $v_{5}$ & $4.895 \cdot 10^{-6}$ \\
$E_{6}$ & $9.376 \cdot 10^{-3}$ & $v_{6}$ & $2.412 \cdot 10^{-8}$ \\
$E_{7}$ & $1.913 \cdot 10^{-6}$ & $v_{7}$ & $2.415 \cdot 10^{-11}$ \\
$E_{8}$ & $1.530 \cdot 10^{-10}$ & $v_{8}$ & $9.867 \cdot 10^{-14}$ \\
$E_{9}$ & $5.488 \cdot 10^{-12}$ & $v_{9}$ & $2.886 \cdot 10^{-15}$ \\
$E_{10}$ & $8.690 \cdot 10^{-11}$ & $v_{10}$ & $2.762 \cdot 10^{-15}$ \\
\hline
\end{tabular}

enhance the basis, which would be desirable as pointed out in the selection criterion (32). This is shown both in the results in Table II and in Fig. 6, where the frequency points selected disagree with the ones suggested by the linear independence criterion (32) detailed in Table I and in Fig. 3. In particular, it is observed that the linear independence measure $\mu_{B_{n}}$ for solution vectors $E_{7}, E_{8}$, and $E_{9}$ is higher for the nonpractical optimal algorithm in Table I than for Algorithm 2. In the same token, it should be noted that $\mu_{B_{8}}\left(E_{9}\right)<\mu_{B_{9}}\left(E_{10}\right)$ in Table II, which shows once again that the greedy algorithm based on the residual error (40) does not ensure to choose the most linearly independent field solutions to uplift the basis. However, it is a cheap and good enough criterion to guide us in the reduced-basis space identification.

Fig. 7 compares the scattering parameter response obtained by this approach with an ROM of order 8 , i.e., reduced-basis space dimension $N=8$, with the one obtained via FEM analysis, where the size of the problem is $\mathcal{N}=36426$. Good agreement is achieved. Taking $10^{-6}$ as linear independence threshold is enough to ensure convergence in the reduced-basis approximation. It should be pointed out that the ROM response is obtained by solving a system matrix of size $N=8$, whereas the full-wave FEM response is computed by solving for large FEM system matrices of size $\mathcal{N}=36426$.

Finally, Table III compares the results achieved by the proposed procedure to the model-order reduction results obtained by means of POD. It should be pointed out that in order to

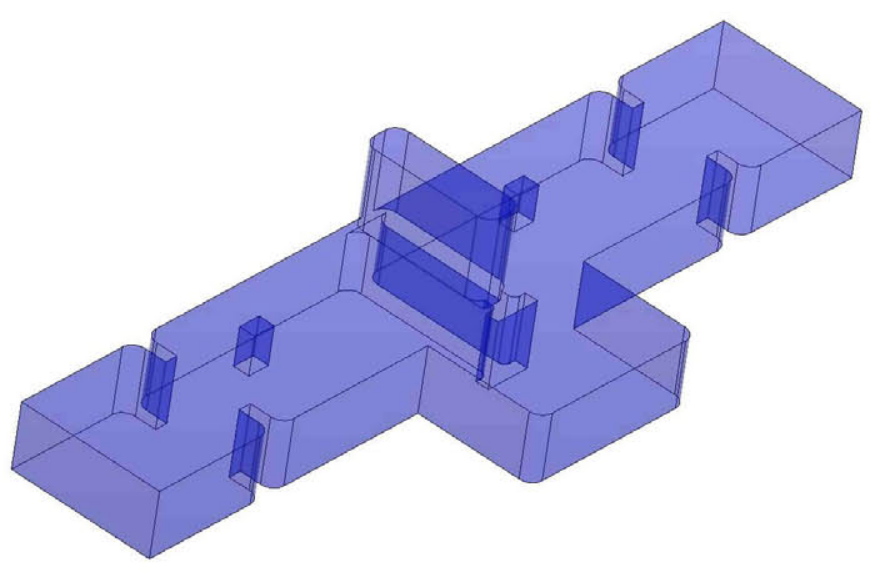

Fig. 8. Geometry of the rectangular waveguide filter designed in [36].

carry out the POD analysis, a total of 1001 evenly spaced electromagnetic-field solutions are computed by direct FEM analysis in the 11.5-12-GHz band. An SVD analysis for all these FEM solutions is carried and the corresponding normalized singular values are detailed in Table III. It should be noted that an ROM of order 6 , i.e., $N_{\mathrm{POD}}=6$, is obtained by POD taking $10^{-6}$ as singular-value threshold, which again is enough to ensure that the POD ROM is accurate. In this POD setting, the reduced-order projection space is provided by the first $N_{\mathrm{POD}}=6$ singular vectors. This optimal result should be compared with the order $N=8$ obtained by the proposed methodology. As a result, we can feel confident that we are proposing a compact basis for reduced basis approximation.

\section{B. Rectangular-Waveguide Filter}

Fig. 8 depicts the geometry of a fifth-order WR-90 rectangular waveguide filter designed in [36] to show the possibility of introducing additional transmission zeros in the filter response. Detailed geometry dimensions can be found in [36]. Since a fifth-order filter is designed, five eigenresonances and the corresponding eigenmodes are expected to be found in the filter operation frequency band.

We carry out a model-order reduction in the $9.2-10.8-\mathrm{GHz}$ band in order to obtain a fast frequency sweep in the filter response. Solving for the eigenmodes in this band, we find the following eigenresonances: $f_{1}=9.766 \mathrm{GHz}, f_{2}=$ $9.812 \mathrm{GHz}, f_{3}=9.944 \mathrm{GHz}, f_{4}=10.075 \mathrm{GHz}$, and $f_{5}=10.157 \mathrm{GHz}$. Then, in order to complete this eigenbasis $B_{5}=\left\{e_{1}, \ldots, e_{5}\right\}$, we proceed as in Algorithm 2. Fig. 9 shows the residual error frequency behavior as we uplift the eigenbasis, and Table IV details the linearly independent information in each of the new field solutions added to the previously built basis.

As a result, an ROM of size $N=10$ is enough to accurately describe the original system response in the $9.2-10.8-\mathrm{GHz}$ band. Again, taking $10^{-6}$ as linear independence threshold is enough to ensure convergence in the reduced-basis approximation. Scattering parameter results obtained by this ROM are compared with FEM analysis in Fig. 10. Good agreement is found. It should be noted that the reduced-basis method 
TABLE IV

LINEAR INDEPENDENCE IN THE FIELD SOLUTIONS IN THE RECTANGULAR WAVEGUIDE FILTER

\begin{tabular}{c|c|c|c}
\hline Frequency $(\mathrm{GHz})$ & Field & Basis & $\mu_{B_{n}}\left(E_{n+1}\right)$ \\
\hline \hline 9.766 & $e_{1}$ & - & - \\
9.812 & $e_{2}$ & $B_{1}$ & 1. \\
9.944 & $e_{3}$ & $B_{2}$ & 1. \\
10.075 & $e_{4}$ & $B_{3}$ & 1. \\
10.157 & $e_{5}$ & $B_{4}$ & 1. \\
10.800 & $E_{6}$ & $B_{5}$ & 0.8528 \\
9.200 & $E_{7}$ & $B_{6}$ & 0.3126 \\
10.025 & $E_{8}$ & $B_{7}$ & $6.263 \cdot 10^{-5}$ \\
10.515 & $E_{9}$ & $B_{8}$ & $1.710 \cdot 10^{-5}$ \\
9.675 & $E_{10}$ & $B_{9}$ & $1.519 \cdot 10^{-7}$ \\
\hline
\end{tabular}

TABLE V

POD SINGULAR-VALUE COMPARISON IN THE RECTANGULAR WAVEGUIDE FILTER

\begin{tabular}{c|c|c|c}
\hline Field & $\mu_{B_{n}}\left(E_{n+1}\right)$ & Singular vector & Singular value \\
\hline \hline$e_{1}$ & - & $v_{1}$ & 1 \\
$e_{2}$ & 1. & $v_{2}$ & 0.01454 \\
$e_{3}$ & 1. & $v_{3}$ & $1.280 \cdot 10^{-3}$ \\
$e_{4}$ & 1. & $v_{4}$ & $4.351 \cdot 10^{-3}$ \\
$e_{5}$ & 1. & $v_{5}$ & $8.008 \cdot 10^{-3}$ \\
$E_{6}$ & 0.8528 & $v_{6}$ & $4.873 \cdot 10^{-5}$ \\
$E_{7}$ & 0.3126 & $v_{7}$ & $2.600 \cdot 10^{-6}$ \\
$E_{8}$ & $6.263 \cdot 10^{-5}$ & $v_{8}$ & $9.999 \cdot 10^{-9}$ \\
$E_{9}$ & $1.710 \cdot 10^{-5}$ & $v_{9}$ & $3.158 \cdot 10^{-10}$ \\
$E_{10}$ & $1.519 \cdot 10^{-7}$ & $v_{10}$ & $3.184 \cdot 10^{-12}$ \\
\hline
\end{tabular}

response is obtained by solving a system matrix of size $N=10$, whereas the FEM response is computed by solving for large FEM system matrices of size $\mathcal{N}=702228$.

Finally, we compare in Table $\mathrm{V}$ the results obtained by the proposed methodology with those achieved by the optimal model-order reduction processing, namely, POD analysis. In order to do so, we compute a total of 321 evenly spaced electromagnetic-field solutions by FEM analysis in the $9.2-10.8-\mathrm{GHz}$ band. Then, an SVD analysis for all these FEM solutions is carried and the corresponding normalized singular values are detailed in Table V. It should be pointed that an ROM of order 8 , i.e., $N_{\mathrm{POD}}=8$, is obtained by POD setting $10^{-6}$ as singular-value threshold, which is enough to ensure that the POD ROM is reliable. In this POD analysis, the reduced-order projection space is spanned by the first $N_{\mathrm{POD}}=8$ singular vectors. This optimal result is compared to the order $N=10$ obtained by the proposed approach. This shows that we are building up a compact basis for reduced-basis approximation without the need to go through any time-consuming POD analysis.

\section{Waveguide Diplexer}

An H-plane WR-90 rectangular waveguide diplexer is studied in this section. This diplexer has been designed in [35] and its geometry is depicted in Fig. 11. This diplexer uses

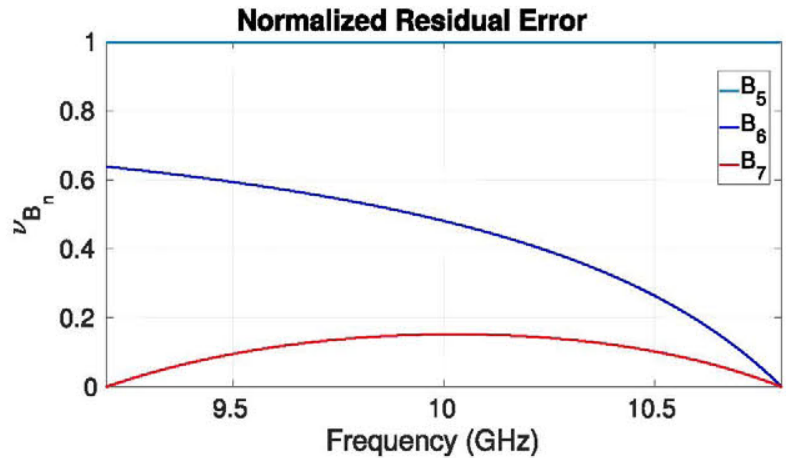

(a)

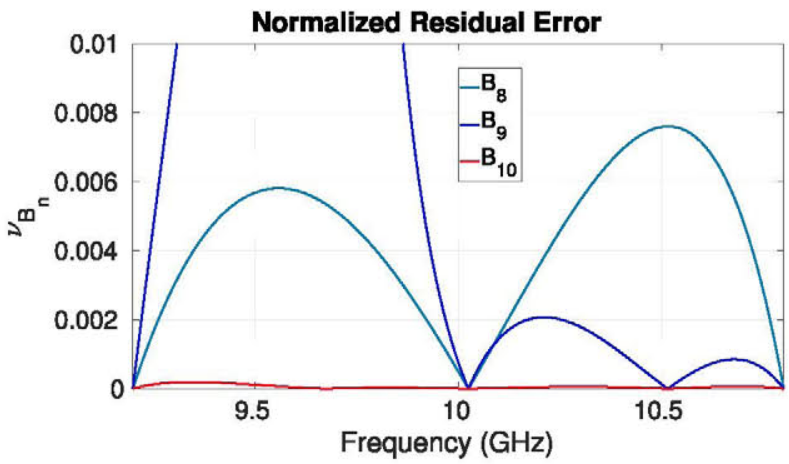

(b)

Fig. 9. Normalized residual error frequency behavior in the reduced-basis approximation as the reduced-basis space is uplifted following the approach detailed in Algorithm 2 in the rectangular-waveguide filter. (a) Normalized residual error in the reduced-basis space spanned by the eigenbasis $B_{5}$ and bases $B_{6}$ and $B_{7}$. A large drop in the residual error is observed as soon as the eigenbasis $B_{5}$ is completed. (b) Normalized residual error in the reduced-basis space spanned by bases $B_{8}, B_{9}$, and $B_{10}$.

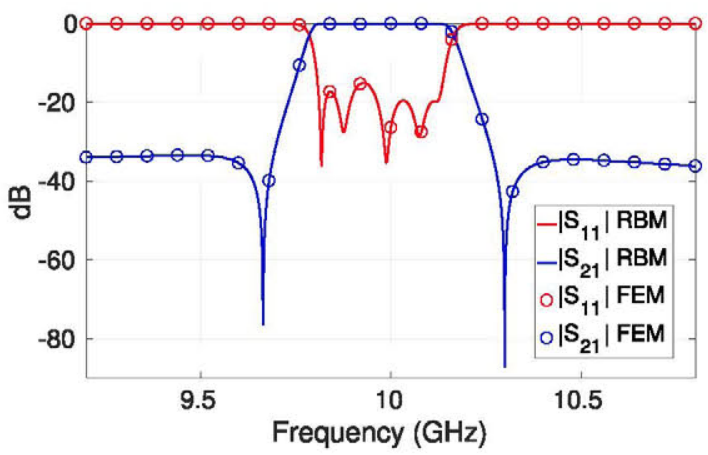

Fig. 10. Rectangular-waveguide filter ROM results are compared with the full-wave FEM scattering parameter response.

two eighth-order filter channels connected by means of a T-junction. At least 16 eigenresonances are expected to be found in the diplexer band. Additional eigenresonances may appear in the diplexer band as a consequence of the T-junction.

The 9-11-GHz frequency band is taken into for fast frequency sweep model-order reduction. Solving for the eigenmodes in this band, we find 18 eigenresonances. This gives rise to an eigenbasis of 18 orthogonal vectors, namely, $B_{18}=$ $\left\{e_{1}, \ldots, e_{18}\right\}$. This eigenbasis is uplifted following the proposed reliable reduced-basis approximation algorithm. The linear independence of each electromagnetic field added to 


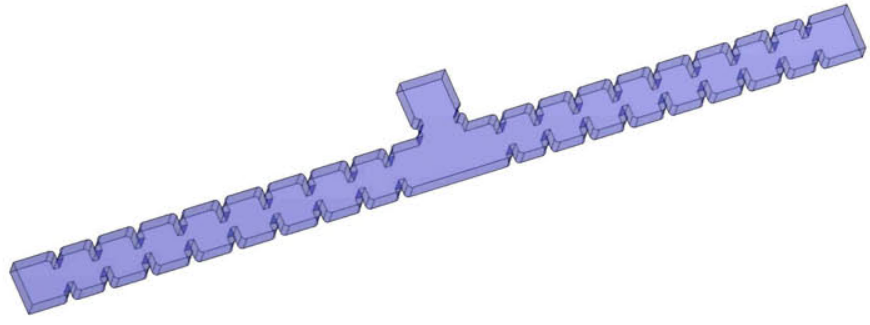

Fig. 11. H-plane rectangular-waveguide diplexer geometry.

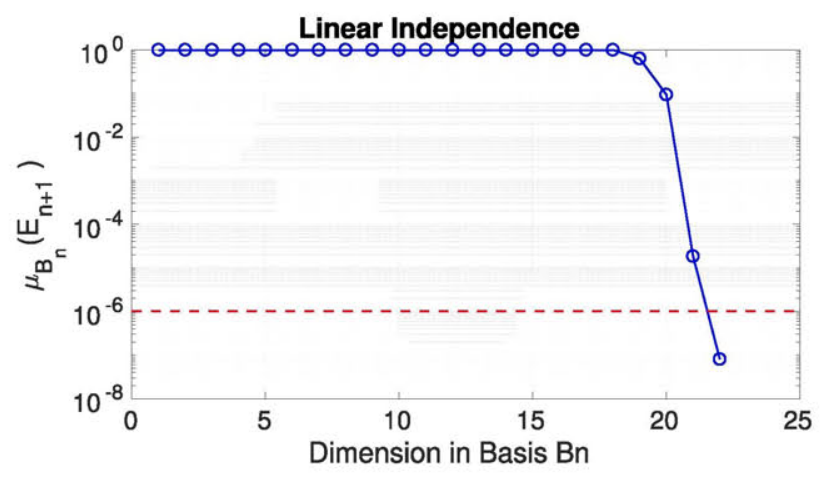

Fig. 12. Linear independence in the field solutions in the waveguide diplexer.

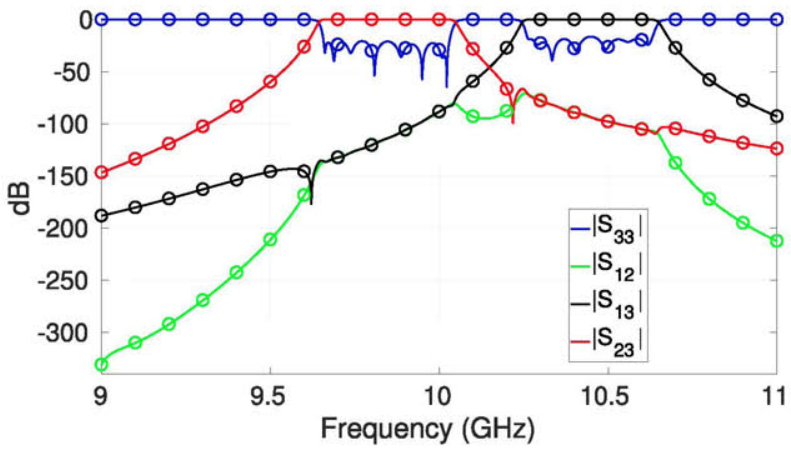

Fig. 13. H-plane rectangular waveguide diplexer ROM results are compared with full-wave FEM scattering parameter response. -: reduced-basis method. ०: FEM.

the basis is detailed in Fig. 12 and the model-order reduction is stopped whenever $10^{-6}$ linear independence threshold is achieved, which ensures convergence in the reduced-basis approximation. As a result, a reduced-basis space of dimension $N=22$ is used for fast frequency sweep in this structure. The results obtained by the ROM are compared with FEM analysis results in Fig. 13. Good agreement between these results is achieved.

\section{Bethe Hole Waveguide Coupler}

Finally, we are going to study a circuit that is no longer a resonating structure operating in a narrowband but a wideband nonresonating circuit, namely, a Bethe hole waveguide coupler. A 4-port microwave circuit is considered this time. This waveguide coupler is detailed in [37] and a design where four holes connecting both WR-90 rectangular waveguide sections is considered. The structure layout as well as its geometric

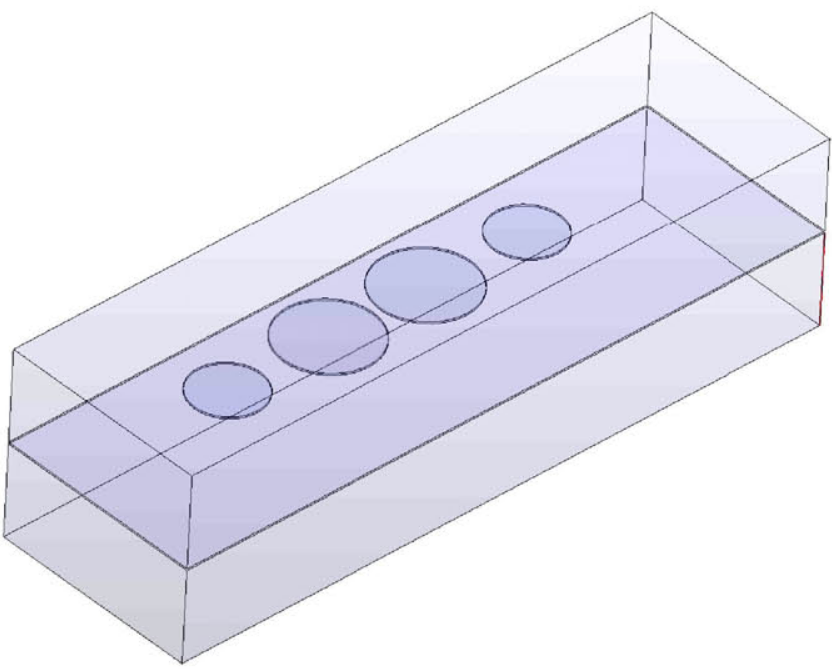

Fig. 14. Bethe hole waveguide coupler geometry. The holes are centered along $1 / 3.5$, the width of WR-90 waveguide. The thickness in the holes is $0.2 \mathrm{~mm}$. The holes are symmetrically inserted along the longitudinal direction in the rectangular waveguides. The radius of the first and the last hole is $3.838 \mathrm{~mm}$. The radius of the middle holes is $5.253 \mathrm{~mm}$. The distance between centers in the first and second holes is $11.807 \mathrm{~mm}$. The distance between centers in the second and the third hole is $11.4 \mathrm{~mm}$.

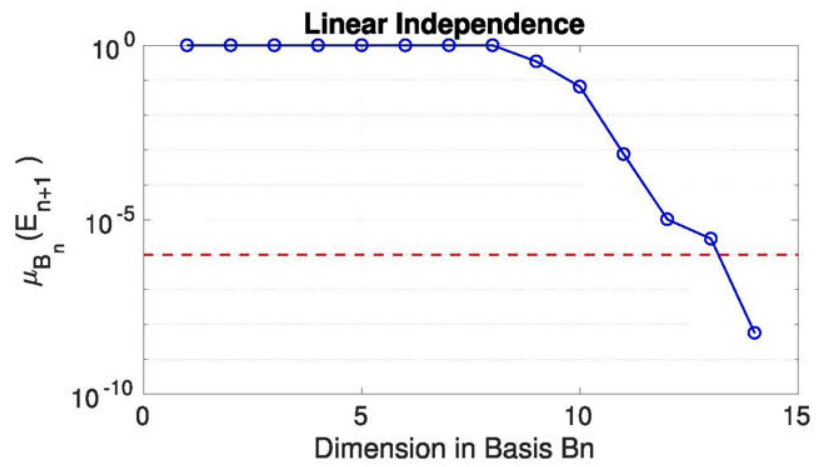

Fig. 15. Linear independence in the field solutions in the waveguide coupler.

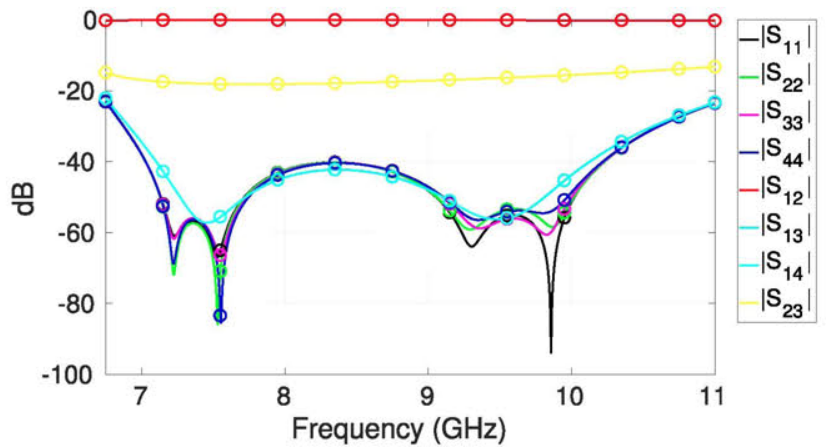

Fig. 16. Bethe hole waveguide coupler ROM results are compared to full-wave FEM scattering parameter response. -: reduced-basis method. $\circ$ : FEM.

dimensions are shown in Fig. 14. A model-order reduction process is carried out in the $6.75-11-\mathrm{GHz}$ band. A total amount of eight eigenresonances are found in the frequency band of interest, yielding an eigenbasis of dimension 8 , namely, $B_{8}=\left\{e_{1}, \ldots, e_{8}\right\}$. This eigenbasis is uplifted by 
the proposed approach until the last field solution added to the reduced-basis space overcomes the linear independence threshold $10^{-6}$. In this circuit, this gives rise to a reduced-basis space of dimension $N=14$ in order to carry out a reliable reduced-basis approximation in the frequency band of interest. Linear independence results are shown in Fig. 15.

Finally, model-order reduction results are compared with FEM in Fig. 16. Good agreement is observed.

\section{CONCLUSION}

An ROM for reliable fast frequency sweep in microwave circuits has been detailed. A compact basis to carry out a reduced-basis approximation has been proposed. Eigenresonances hit in the frequency band of interest have been no longer avoided but considered in the reduced-basis method. As a result, a reduced-basis space spanned by eigenmodes, giving rise to an eigenbasis, and additional adaptively selected electromagnetic fields, has been addressed. All these eigenmodes and electromagnetic fields have been appropriately chosen within the frequency band of interest.

A special emphasis has been considered in certifying the accuracy in the model-order reduction as well as providing a reliable stopping criterion in the construction of the ROM, based on the linear independence in the electromagnetic fields adaptively chosen to complete the eigenbasis in the reduced-basis space.

Several numerical results including both narrowband resonating structures and wide-band non-resonating devices have shown the capabilities and reliability of the proposed approach. It should be pointed out that the proposed methodology has performed as good as POD in terms of the ROM order throughout the examples where POD analysis has been considered.

Radiation problems can also be considered within the proposed methodology by introducing radiating ports. In this setting, the field at the open boundary is described in terms of spherical modes. This approach has already been detailed in [22].

In addition, the proposed methodology can be further applied to lossy microwave devices. It has to be pointed out that in the case of lossy media, the eigenresonances are complex, but still eigenmodes can be found associated to these complex eigenresonances. These give rise to the eigenbasis that is further used in the reduced-basis approximation.

Let us point out that we have aimed at providing a compact basis for reduced-basis approximations. In terms of computational efficiency, the method we have proposed may or may not be faster than other practical model-order reduction techniques, such as SVD or moment-matching approaches. This depends on the specific computing scenario. For instance, if the FEM system matrix is too large to be factorized, other reduction techniques will be faster, but in case the FEM system matrix is not too large to be factorized and the frequency band is not too large as well, we expect our methodology not only to provide a compact basis, but also to be faster than other model-order reduction approaches.

We have shown that the reduced-basis method we have proposed has allowed to model the electromagnetic behavior of a circuit by solving a very small system of equations, of size $N$, the order of the ROM. However, this reduction has not come for free, since the reduced-basis method does need to build a reduced-basis space up first in order to compute the ROM. This computation has been carried out offline and does have required a few FEM analyses, namely $N$, the order of the ROM. Specifically, it has involved $N-K+1$ sparse matrix factorizations, where $K$ is the number of eigenmodes hit in the frequency band of interest. Note that one sparse matrix factorization is used to solve for the eigenvalues and eigenmodes in the frequency band of interest.

\section{REFERENCES}

[1] A. C. Antoulas, Approximation of Large-Scale Dynamical Systems. Philadelphia, PA, USA: SIAM, 2005.

[2] P. Feldmann and R. W. Freund, "Efficient linear circuit analysis by Pade approximation via the Lanczos process," IEEE Trans. Comput.-Aided Design Integr. Circuits Syst., vol. 14, no. 5, pp. 639-649, May 1995.

[3] J. Rubio, J. Arroyo, and J. Zapata, "SFELP-an efficient methodology for microwave circuit analysis," IEEE Trans. Microw. Theory Techn., vol. 49, no. 3, pp. 509-516, Mar. 2001.

[4] L. T. Pillage and R. A. Rohrer, "Asymptotic waveform evaluation for timing analysis," IEEE Trans. Comput.-Aided Design Integr. Circuits Syst., vol. 9, no. 4, pp. 352-366, Apr. 1990.

[5] R. D. Slone, R. Lee, and J.-F. Lee, "Well-conditioned asymptotic waveform evaluation for finite elements," IEEE Trans. Antennas Propag., vol. 51, no. 9 , pp. 2442-2446, Sep. 2003.

[6] D. Jiao, J. Zhu, and S. Chakravarty, "A fast frequency-domain eigenvalue-based approach to full-wave modeling of large-scale threedimensional on-chip interconnect structures," IEEE Trans. Adv. Packag., vol. 31 , no. 4, pp. 890-899, Nov. 2008.

[7] T. Wittig, R. Schuhmann, and T. Weiland, "Model order reduction for large systems in computational electromagnetics," Linear Algebra Appl., vol. 415, nos. 2-3, pp. 499-530, 2006.

[8] B. Stupfel, "Frequency interpolation of the electromagnetic surface currents via singular value decomposition. Application to highfrequency analysis," IEEE Trans. Antennas Propag., vol. 56, no. 12, pp. 3789-3796, Dec. 2008.

[9] M. Rewienski, A. Lamecki, and M. Mrozowski, "Greedy multipoint model-order reduction technique for fast computation of scattering parameters of electromagnetic systems," IEEE Trans. Microw. Theory Techn., vol. 64, no. 6, pp. 1681-1693, Jun. 2016.

[10] G. Fotyga, M. Czarniewska, A. Lamecki, and M. Mrozowski, "Reliable greedy multipoint model-order reduction techniques for finiteelement analysis," IEEE Antennas Wireless Propag. Lett., vol. 17, no. 5, pp. 821-824, May 2018.

[11] A. Hochman, J. F. Villena, A. G. Polimeridis, L. M. Silveira, J. K. White, and L. Daniel, "Reduced-order models for electromagnetic scattering problems," IEEE Trans. Antennas Propag., vol. 62, no. 6, pp. 3150-3162, Nov. 2011.

[12] Q. I. Dai, Y. H. Lo, W. C. Chew, Y. G. Liu, and L. Jiang, "Generalized modal expansion and reduced modal representation of 3-D electromagnetic fields," IEEE Trans. Antennas Propag., vol. 62, no. 2, pp. 783-793, Feb. 2014

[13] J. Rommes and N. Martins, "Efficient computation of transfer function dominant poles using subspace acceleration," IEEE Trans. Power Syst., vol. 21, no. 3, pp. 1218-1226, Aug. 2006.

[14] F. D. Freitas, J. Rommes, and N. Martins, "Gramian-based reduction method applied to large sparse power system descriptor models," IEEE Trans. Power Syst., vol. 23, no. 3, pp. 1258-1270, Aug. 2008.

[15] S.-H. Lee and J. M. Jin, "Efficient full-wave analysis of multilayer interconnection structures using a novel domain decomposition-modelorder reduction method," IEEE Trans. Microw. Theory Techn., vol. 56, no. 1, pp. 121-129, Jan. 2008.

[16] O. Floch, A. Sommer, D. Klis, O. Farle, and R. Dyczij-Edlinger, "An adaptive deflation domain-decomposition preconditioner for fast frequency sweeps," IEEE Trans. Magn., vol. 52, no. 3, Mar. 2016, Art. no. 7200504

[17] W. Wang, G. N. Paraschos, and M. N. Vouvakis, "Fast frequency sweep of FEM models via the balanced truncation proper orthogonal decomposition," IEEE Trans. Antennas Propag., vol. 59, no. 11, pp. 4142-4154, Nov. 2011. 
[18] R. Baltes, A. Schultschik, O. Farle, and R. Dyczij-Edlinger, "A finiteelement-based fast frequency sweep framework including excitation by frequency-dependent waveguide mode patterns," IEEE Trans. Microw. Theory Techn., vol. 65, no. 7, pp. 2249-2260, Jul. 2017.

[19] M. Jemai and A. B. Kouki, "New adaptive multi-expansion frequencies approach for SP-MORe techniques with application to the wellconditioned asymptotic waveform evaluation," IEEE Trans. Microw. Theory Techn., vol. 65, no. 10, pp. 3709-3719, Oct. 2017.

[20] A. Quarteroni, A. Manzoni, and F. Negri, Reduced Basis Methods for Partial Differential Equations. Cham, Switzerland: Springer, 2016.

[21] J. S. Hesthaven, G. Rozza, and B. Stamm, Certified Reduced Basis Methods for Parametrized Partial Differential Equations. Berlin, Germany: Springer, 2016.

[22] V. de la Rubia, U. Razafison, and Y. Maday, "Reliable fast frequency sweep for microwave devices via the reduced-basis method," IEEE Trans. Microw. Theory Techn., vol. 57, no. 12, pp. 2923-2937, Dec. 2009.

[23] M. Rewienski, A. Lamecki, and M. Mrozowski, "A goal-oriented error estimator for reduced basis method modeling of microwave devices," IEEE Microw. Wireless Compon. Lett., vol. 25, no. 4, pp. 208-210, Apr. 2015.

[24] A. Sommer, O. Farle, and R. Dyczij-Edlinger, "A new method for accurate and efficient residual computation in adaptive model-order reduction," IEEE Trans. Magn., vol. 51, no. 3, Mar. 2015, Art. no. 1100104.

[25] M. W. Hess and P. Benner, "Fast evaluation of time-harmonic Maxwell's equations using the reduced basis method," IEEE Trans. Microw. Theory Techn., vol. 61, no. 6, pp. 2265-2274, Jun. 2013.

[26] M. W. Hess, S. Grundel, and P. Benner, "Estimating the inf-sup constant in reduced basis methods for time-harmonic Maxwell's equations," IEEE Trans. Microw. Theory Techn., vol. 63, no. 11, pp. 3549-3557, Nov. 2015.

[27] S. García, V. de la Rubia, and M. Mrozowski, "Reduced basis approximations in microwave filters and diplexers: Inf-sup constant behavior," in IEEE MTT-S Int. Microw. Symp. Dig., May 2017, pp. 275-277.

[28] V. Girault and P.-A. Raviart, Finite Element Methods for Navier-Stokes Equations. Berlin, Germany: Springer-Verlag, 1986.

[29] P. Monk, Finite Element Methods for Maxwell's Equations. New York, NY, USA: Oxford Univ. Press, 2003.

[30] K. Yosida, Functional Analysis. Berlin, Germany: Springer, 1980.

[31] W. McLean, Strongly Elliptic Systems and Boundary Integral Equations. Cambridge, U.K.: Cambridge Univ. Press, 2000.

[32] R. Kress, Linear Integral Equations. Berlin, Germany: Springer, 1999.

[33] A. Kirsch and F. Hettlich, The Mathematical Theory of Time-Harmonic Maxwell's Equations. Cham, Switzerland: Springer, 2015.
[34] K. Kurokawa, An Introduction to the Theory of Microwave Circuits. New York, NY, USA: Academic, 1969.

[35] A. Lamecki, L. Balewski, and M. Mrozowski, "An efficient framework for fast computer aided design of microwave circuits based on the higherorder 3D finite-element method," Radioengineering, vol. 23, no. 4, pp. 970-978, 2014.

[36] L. Szydlowski and M. Mrozowski, "A self-equalized waveguide filter with frequency-dependent (resonant) couplings," IEEE Microw. Wireless Compon. Lett., vol. 24, no. 11, pp. 769-771, Nov. 2014.

[37] D. M. Pozar, Microwave Engineering. New York, NY, USA: Wiley, 1998 , ch. 7.

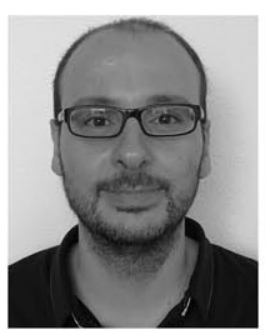

Valentín de la Rubia received the Ingeniero de Telecomunicación and Ph.D. degrees from the Universidad Politécnica de Madrid, Madrid, Spain, in 2003 and 2008, respectively.

He was an Assistant Professor with the Universidad de Oviedo, Asturias, Spain, from 2007 to 2008, and with the Universidad de Extremadura, Extremadura, Spain, from 2008 to 2011. Since 2011, he has been an Assistant Professor with the Universidad Politécnica de Madrid.

His current research interests include computational electromagnetics and model-order reduction techniques for microwave circuit and antenna design.

Michal Mrozowski (S'88-M'90-SM'02-F'08) received the M.Sc. and Ph.D. degrees from the Gdańsk University of Technology, Gdańsk, Poland, in 1983 and 1990, respectively.

In 1986, he joined the Faculty of Electronics, Gdańsk University of Technology, where he is currently a Full Professor and also the Head of the Department of Microwave and Antenna Engineering. His current research interests include computational electromagnetics, CAD of microwave devices, filter design, and optimization techniques. 